\title{
The dynamics of inequalities and unequal exchange of labor in intertemporal linear economies*
}

\author{
Giorgos Galanis ${ }^{\dagger}$ \\ Roberto Veneziani ${ }^{\ddagger}$ \\ Naoki Yoshihara ${ }^{\S}$
}

December 3, 2018

\begin{abstract}
Introducing a concept of fairness of economic allocations, namely exploitation as the unequal exchange of labor (henceforth, UE exploitation) by generalizing Roemer's [51, 52] seminal model, this paper aims to answer the following two questions in the context of an intertemporal economy with linear technology: How is income and wealth inequality related (or unrelated) to the existence and persistence of UE exploitation? What are the mechanisms driving the persistent existence of UE exploitation in growing economies? Agents are UE exploited (resp. exploiters) if the amount of labor that they contribute to the economy is smaller (resp. bigger) than the amount of labor 'received' by them via their income. It is proved, first, that UE exploitation is monotonically correlated to functional income inequality. Second, it is shown that, unless agents discount the future, asset inequalities are necessary, but not sufficient for the persistence of UE exploitation, and the capital accumulation leading to the disappearance of UE exploitation cannot be ruled out in equilibrium. Third, it is shown that, regardless of whether agents discount the future, labor-saving technical progress may yield sustained growth with persistent UE exploitation by keeping labor abundant relative to capital, which restrains wages from rising. Unlike in models with differentiable production functions, this mechanism does not rely on changes in the marginal productivity of inputs and it is entirely driven by the interaction between innovation and labor markets.
\end{abstract}

JEL classification: D51; D63; C61; E11.

Keywords: Dynamics, accumulation, exploitation, inequalities.

\footnotetext{
${ }^{*}$ We are grateful to Jon Cogliano, Meghnad Desai, Amitava K. Dutt, Peter Flaschel, Peter H. Matthews, John Roemer, Peter Skott, Robert Sugden, Andrew Trigg, Alessandro Vercelli, and audiences in New York, London, Bristol, and Cambridge for comments and suggestions on an early draft ("Accumulation and exploitation in a Linear Economy"). Special thanks go to Gil Skillman, three anonymous referees and the Editors of this Journal for long and detailed comments. The usual disclaimer applies.

${ }^{\dagger}$ Institute of Management Studies, Goldsmiths, University of London, New Cross, London SE14 6NW, U.K, E-mail: g.galanis@gold.ac.uk

$¥$ (Corresponding author) School of Economics and Finance, Queen Mary University of London, Mile End Road, London E1 4NS, U.K. E-mail: r.veneziani@qmul.ac.uk

${ }^{\S}$ Department of Economics, University of Massachusetts Amherst, Crotty Hall, Amherst, MA, 01002, USA; The Institute of Economic Research, Hitotsubashi University, Naka 2-1, Kunitachi, Tokyo 186-0004, Japan; and School of Management, Kochi University of Technology, Tosayamada, Kami-city, Kochi 782-8502, Japan, E-mail: nyoshihara@econs.umass.edu.
} 


\section{Introduction}

Recently, a vast literature has analyzed the persistent, and widening, inequalities in income and wealth observed in the vast majority of nations. ${ }^{1}$ Less attention has been devoted to a specific form of inequality related to the systematic underpayment of labor in relation to their contribution to production, which is known as exploitation and has normative implications. Two questions immediately arise. First, what is the relation between inequalities in income and/or wealth, and the existence and persistence of exploitation? Second, what are the mechanisms that drive the existence, and persistence, of exploitation in growing economies?

The answers to these questions are not obvious, and depend on the concept of exploitation adopted. ${ }^{2}$ This paper adopts a specific concept of exploitation, namely exploitation as the unequal exchange of labor (henceforth, UE exploitation), and analyzes the relation between inequalities in income and/or wealth, and the existence and persistence of UE exploitation by focusing on linear economies. ${ }^{3}$ The basic intuition of UE exploitation can be traced back to Aristotle and Karl Marx, ${ }^{4}$ but its modern, rigorous formulation is due to John Roemer [50, 52, 53]: an agent is UE exploited (resp. exploiting) if the amount of labor she contributes to the economy (e.g., the labor she expends in productive activities) is higher (resp. lower) than the amount of labor she 'receives' via her income (e.g. the labor contained in the consumption bundle she can purchase). As Roemer ([53], p. 168) emphasized, this kind of disproportional distribution of labor and national income is indeed worth calling exploitative under the differential ownership of productive assets.

To see this, consider a simple perfectly competitive economy with only two agents, $\nu, \mu$ with the same preferences and the same skills. If agent $\nu$ is wealthy and therefore can optimize without working, while agent $\mu$ has very little capital and needs to work for $\mu$, then "[p]roducer $\nu$ is exploiting $\mu$. This comes about because $\ldots \nu$ is wealthier than $\mu$, and is able to use his wealth as leverage through the exchange mechanism to force $\mu$ to work "for" him. ... That this phenomenon deserves to be called [UE] exploitation can be seen by the following. Suppose $\ldots \nu$ expropriated $\mu$ 's endowment and killed him. Producer $\nu$ would now be wealthier than before; yet, ... for the new economy in which only he is a member, he will have to work ... longer than when $\mu$ was there. Thus, exploitation is an explicitly social phenomenon: $\nu$ can get away with working less ... only because there is someone else working more ..., to "support" him. Producer $\nu$ appears to be gaining at the expense of $\mu, \ldots$ even though all producers gain from trade" (Roemer [53], p.168).

This last argument also makes the concept of UE exploitation distinct from those of the other forms of inequalities. Indeed, if these agents engaged in their autarkic economic activities and developed no division of labor between them, income and wealth inequalities would be still observed due to the differential ownership of productive assets, but there is no UE exploitation in such a case.

The concept of UE exploitation also directs our attention to the joint distribution of income

\footnotetext{
${ }^{1}$ For example see [44].

${ }^{2}$ One example of such a concept is of "neoclassical exploitation" which can be traced back to Arthur Pigou [43] and Joan Robinson [49], according to which exploitation occurs when the rental price of a productive input is not equal to its marginal product. Therefore, exploitative relations arise from competitive distortions and so exploitation persists for as long as such distortions persist. Thus, first, if marginal product pricing obtains, then voluntary transactions in factor markets are automatically fair, whatever the background conditions of (and the final income distribution resulting from) such trades. Second, and more important, exploitation cannot be diagnosed (and therefore the concept has no bite) whenever the marginal product of a factor of production cannot be defined. In particular, the concept cannot be used in the large, and theoretically relevant set of linear economies, in which all income distributions are definitionally, and rather counterintuitively, nonexploitative.

${ }^{3}$ The focus on linear economies is empirically in line with recent evidence casting doubts on the degree of substitutability between labor and capital, and showing some limitations of marginal productivity theory as an explanation of the remuneration of productive inputs (Foley and Michl [17]; Basu [8]).

${ }^{4}$ For a discussion, see Roemer [52], Maniquet [34], and Yoshihara and Veneziani [69]. The concept of UE exploitation is also related to the so-called "proportional solution" and the notion of Kantian equilibrium (Roemer and Silvestre [56]; Moulin [39]; Roemer [55]).
} 
and labor/leisure in the economy, which is also relevant information for the modern theory of distributive justice. Indeed, as Fleurbaey [16] has argued, UE exploitation can be linked with the issue of inequalities in the distribution of material well-being purchasable via income (ex. goods, services, etc.) and free hours (leisure time) discussed in the theory of distributive justice. For instance, they are relevant for inequalities of well-being freedom ${ }^{5}$ (Rawls [48]; Sen [57]), because material well-being and free hours are key determinants of individual well-being freedom.

Given these distinctive features of the concept of UE exploitation, the first question raised at the head of this section is important in order to understand whether analyses of income and wealth inequalities provide exhaustive information about the unfairness of competitive economies. If this was not the case, then policies that alleviate inequalities would not necessarily reduce, and might even increase, other forms of unfairness, such as UE exploitation.

The relation between growth and UE exploitation is also unclear, and equally relevant from both a theoretical and a policy perspective. Does growth tend to reduce, or worsen, exploitative relations? What are the mechanisms that guarantee the persistence of UE exploitation?

In this paper, we address these issues and analyze the relation between wealth inequalities, profits, accumulation and UE exploitation in a dynamic general equilibrium model with heterogeneous optimizing agents which generalizes Roemer's $[50,52,53]$ economies. To be specific, we assume that there exist two types of agents in the economy, capitalists and workers. Both types of agents have the same preferences over consumption bundles and solve an intertemporal optimization program. They also have the same skills and labor endowments. However, we allow for inequalities in their endowments of productive assets, which have some major behavioral implications.

Capitalists own wealth which allows them to hire workers and activate production processes by using a linear technology. They do not work and allocate their income optimally between consumption and savings. In contrast, workers own no wealth and their only source of income is their labor. Although, like capitalists, they choose consumption bundles to maximize utility, we assume that they cannot accumulate. This assumption is consistent with two other key features of our model, namely the existence of wealth constraints and the incompleteness of credit markets, ${ }^{6}$ whose importance has long been emphasized in the literature. ${ }^{7}$ It is also in line with standard models in the tradition of Classical economics (see Roemer [50], p.509), and with more recent analyses of the effect of wealth inequality and credit constraints on macroeconomic dynamics (see, most notably, Kaplan and Violante [26] and Krueger et al [29]). ${ }^{8}$

In this economy, we rigorously define the concept of UE exploitation (Definition 3): agents suffer from an unequal exchange of labor if the amount of labor they contribute to the economy is higher than the amount of labor necessary to produce a consumption bundle purchased by their income. We show that in equilibrium, the profitability of capitalist production is synonymous with the existence of UE exploitation and a monotonic relation exists between the profit rate ${ }^{9}$ and the exploitation rate (Theorem 1). This result is important because the rate of profit is one of the key determinants of investment decisions, and of the long-run dynamics of capitalist economies. Thus,

\footnotetext{
${ }^{5}$ The notion of well-being freedom emphasizes an individual's ability to pursue the life she values.

${ }^{6}$ In economies with wealth constraints, the availability of production loans is a necessary condition for asset-poor agents to be able to undertake production activities and obtain nonzero and nonegligible wealth levels in equilibrium (Banerjee and Newman [6]).

${ }^{7}$ See, for example, the classic papers by Loury [31]; Galor and Zeira [18]; Bénabou [10]; and Matsuyama [35]).

${ }^{8}$ Empirically, this assumption is consistent with recent findings by Krueger et al [29] who report higher consumption rates for agents with lower net worth. It can also be justified noting the extreme, and persistent, wealth inequalities in advanced economies, with the bottom quintiles of the wealth distribution owning zero net worth (Allegretto [4]; Piketty [44]; Krueger et al [29]), and with remarkably (and perhaps surprisingly) low and declining degree of social mobility (Greenstone et al [21]). This is true even if one considers the very long run (Barone and Mocetti [7]) and without major differences between European countries and the US (Bénabou and Tirole [11], pp.702ff).

${ }^{9}$ We define 'profits' as all incomes other than incomes from labor, or 'unearned incomes' as they are sometimes called, and the rate of profit is the ratio of unearned incomes over the value of the capital employed in production (see Malinvaud ([32], pp.254-255).
} 
Theorem 1 can be interpreted as providing a link between inequality, exploitation, and growth: in an economy where only capitalists invest, more exploitation implies a higher profit rate and thus more funds for accumulation. But the result is important also because it proves that, given private ownership of productive assets, profits are a counterpart of the transfer of social surplus and social labor from asset-poor agents to the wealthy. This provides a link between UE exploitation and the functional income distribution. This result confirms the relevance of the concept of UE exploitation in examining the dynamics of capitalist economies, and it provides the foundations for the rest of the analysis.

We then derive a number of results concerning the relation between inequality, UE exploitation, and growth. First, in the basic economy with constant labor supply and a given technology, there exists no equilibrium path with persistent growth and persistent UE exploitation even in the presence of significant asset inequalities (Proposition 1): absent any countervailing measures, accumulation leads capital to become abundant, and profits and exploitation to disappear. Second, and perhaps more surprising, asset inequalities and competitive markets alone do not guarantee the persistence of profits and UE exploitation, even in equilibrium paths without accumulation in which capital does not become abundant. Neither at stable growth paths converging to a long-run steady state, nor at stationary states without accumulation, is UE exploitation a persistent feature of the economy, unless agents discount the future (Theorems 3 and 4). Finally, Theorem 7 proves that in the long-run labor-saving technical progress may yield sustained growth with persistent exploitation and inequalities by reducing the demand for labor, thus creating the conditions for an excess supply of labor, which restrains wages from rising. ${ }^{10}$

These results correspond to the question of the mechanisms driving the persistent existence of UE exploitation in growing economies. First, a part of them sheds light on the role of time preference in this question. If the discount factor of agents is unity, then inequalities, UE exploitation, and profits may well disappear after a finite number of periods, even if capital remains scarce (Theorem 3 and Theorem 4). Instead, if agents discount the future, inequalities and UE exploitation can be persistent even in paths with capital accumulation (Theorem 4). Furthermore, the steady state value of the profit rate (and thus, by Theorem 1, the rate of UE exploitation) is a positive function of the discount factor whenever it is less than unity. In contrast, overaccumulation leading to the disappearance of UE exploitation cannot be ruled out in equilibrium unless the discount factor is less than unity (Proposition 2). These results also suggest that the analysis of UE exploitation cannot be reduced to the analysis of wealth inequalities, as discussed in more detail at sections 5 and 6 later.

Given these results, one may argue that, along equilibrium paths where capital remains scarce, a positive rate of time preference by capitalists is all that is needed, in addition to differential ownership of (scarce) productive assets and competitive markets, to guarantee the persistence of factor income inequalities and UE exploitation. Although this is certainly a legitimate interpretation of Theorems 3 and 4, we do not think that it is the most insightful or normatively robust. On the one hand, in our view, one of the distinguishing features of exploitation theory is an emphasis on the structural, objective characteristics of capitalist economies, rather than on subjective and empirically contingent factors such as time preference.

On the other hand, our results point to an alternative, more structural explanation of the persistence of inequalities and UE exploitation. In particular, Theorem 7 suggests that the central role of asset inequalities is better understood in conjunction with labor market conditions and institutions, and the mechanisms that ensure the scarcity of capital. It provides an alternative explanation of the persistence of UE exploitation focusing on the interaction between technical change and labor market conditions in shaping distributive outcomes and the equilibrium growth paths of capitalist economies. ${ }^{11}$ By raising labor productivity, labor-saving technical progress

\footnotetext{
${ }^{10}$ For a discussion of the role of technical change and a review of the literature, see Tavani and Zamparelli [61].

${ }^{11}$ This conclusion is similar in spirit to the main findings of Banerjee and Newman [5] where persistent inequalities
} 
ensures the persistent abundance of labor, which in turn keeps wages low and guarantees the profitability conditions necessary for growth. Further, as the wage rate and employment remain relatively stagnant while the economy grows, the interaction between labor market conditions and technical progress leads the wage share in national income to fall steadily over time, as has been observed in most advanced countries in the last few decades (Karabarbounis and Neiman [27]; Piketty and Zucman [45]).

Compared with the recent macroeconomic literature exploring the emergence and persistence of inequality, our approach with a linear production technology is distinctive, in that it allows us to examine some relatively unexplored mechanisms through which inequality affects growth, and vice versa. In economies with credit constraints and standard production functions with diminishing marginal productivity, for example, inequality tends to reduce growth because poorer agents cannot access production loans and therefore cannot invest in projects yielding higher returns (see Bénabou [10]; Matsuyama [35]; and the literature therein). Conversely, one of the main channels through which growth affects inequality is via the effect of accumulation on the dynamics of the marginal productivity of capital and labor. In our model, we analyze the relation between inequality and growth abstracting from any consideration concerning marginal productivity, while focusing on the role of labor market conditions and technical change.

Further, while technical change and capital accumulation are also central in Acemoglu [1, 2, 3], they play a different role in their interaction with labor market conditions. Innovations, in our model, do not allow for persistent growth by affecting total factor productivity, or via their direct effect on output per worker, and they do not influence the functional distribution of income by altering marginal productivity. Rather, labor-saving technical change allows UE exploitation and inequalities to persist by constantly creating an excess supply of labor that weakens the workers' position in the labor market and drives wages down.

\section{Related Literature}

This paper lies at the intersection of various strands of literature.

Roemer's $[50,52,53]$ seminal contributions have sparked a vast debate. Several critiques have been expounded on his methodology and conclusions, mainly based on exegetical issues, but surprisingly little attention has been devoted to his models (Veneziani [62]). In this paper, we take a different approach and critically evaluate Roemer's theory using a dynamic general equilibrium model. For, as Veneziani [62] has argued, Roemer's [50, 52, 53] models are essentially static in that agents face no intertemporal trade-offs. As a result, they are not suitable to analyze exploitation and inequalities as persistent features of capitalist economies. Nor do they allow to analyze the relation between exploitation, inequality and accumulation.

This paper analyzes the conditions both for the emergence and for the persistence of exploitation, and the relation between asset inequalities, exploitation, profits and growth, in a dynamic generalization of Roemer's [50, 52, 53] economies with optimizing agents. This allows us to assess the causal and moral relevance of asset inequalities in generating exploitation as a persistent feature of a capitalist economy where (a subset of) agents can save.

Our approach is reminiscent of earlier contributions by Devine and Dymski [14] and Hahnel [22], who have showed that if Roemer's [52] static model is allowed to run for many periods, capital accumulation eventually drives profits to zero. However, unlike in this paper, they focused on the $T$-fold iteration of the static model with myopic agents, without explicitly analyzing intertemporal decisions. Moreover, they did not explicitly consider the possible determinants of the persistence of exploitation and inequalities. Some of our conclusions echo the results obtained in Veneziani [62]. However, the latter contributions focus on simple subsistence economies with a given technology,

derive from the interaction between labor market conditions and wealth constraints in a model of occupational choice. 
and therefore can only shed partial light on the determinants of exploitation and on the link between exploitation and growth.

The model incorporates a number of assumptions - most notably, concerning labor, technology, savings, and technical change - that can be traced back to the Classical economists, and that allow us "to focus on the effect of ongoing capital accumulation per se on the long run distribution of income between capital and labor" (Mookherjee and Ray [36], p.2). These assumptions have been formalized in some classic papers, such as Kaldor [24] and Pasinetti [41], which focused on the effect of income distribution on consumption and saving and sparked a vast debate (more recent work includes Bohm and Kaas [13] and Patriarca and Vona [42]). Unlike in these earlier contributions, however, we explicitly model micro behavior and intertemporal trade-offs.

There is a vast literature analyzing the effect of inequality on growth focusing on electoral competition and political economy (Bénabou [10]; Ray and Esteban [46]): in these contributions, inequality tends to have a detrimental effect on growth as it generates social conflict and insecure property rights, but "what really matters is not income inequality per se but inequality in the relative distribution of earning and political power" (Bénabou [10], p.18): a higher degree of prowealth bias in the political system tends to help growth. We abstract from political economy issues and in our model the role of capitalists in fostering accumulation implies that inequality always has beneficial effects on growth, consistent with standard insights in Classical economics.

Our results relate to a literature that explores the emergence and persistence of inequality, including human capital and nonconvexities (Galor and Zeira [18]; Galor and Tsiddon [19]), endogenous risk-taking or idiosyncratic and uninsurable unemployment risk (Becker, Murphy, and Werning [9]; Ray and Robson [47]; Krueger et al [29]), and aspirational preferences (Genicot and Ray [20]). Yet our analysis is conceptually closer to contributions taking a more structural perspective and emphasizing the role of incomplete markets, credit constraints, and institutions (Loury [31]; Banerjee and Newman [5]; Matsuyama [35]), and the interaction between labor market conditions and technical change (Acemoglu [1, 2, 3]).

Compared with the literature just mentioned, our approach is distinctive given the focus on UE exploitation and on the relation between exploitation and the functional income distribution. In this respect, our paper is closer to the growing literature analysing the recent trend decline in the labor share both in the U.S. and globally, focusing on technical change and automation (Karabarbounis and Neiman [27]; Mookherjee and Ray [36]) or capital accumulation (Piketty [44]; Piketty and Zucman [45]). Although technical change and capital accumulation are central in our model, they play a different role in their interaction with labor market conditions. Thus, unlike in Karabarbounis and Neiman [27] and Mookherjee and Ray [36], innovations and accumulation do not affect distribution via their effect on the relative price of investment goods. Unlike in Piketty [44] and Piketty and Zucman [45], capital accumulation does not have a negative effect on the wage share and indeed when the conditions for long run accumulation are satisfied the growth rate of the economy is equal to the profit rate.

Our model also relates to the literature on the generic indeterminacy of the functional income distribution in perfectly competitive economies with a linear technology (Mandler [33] and Yoshihara and Kwak [70]). By proving the monotonic relation between UE exploitation and profits in Theorem 1, our paper suggests that exploitative relations between capitalists and workers may serve as a selection mechanism to identify an equilibrium factor income distribution, thus providing the foundations for an alternative to marginal productivity theory.

Finally, Theorem 1 proves that profits are synonymous with UE exploitation. The existence of a relation between exploitation and profits has a "prominent place in the modern formulation of Marxian economics" (Roemer [51], p.16), and therefore it has been dubbed the "Fundamental Marxian Theorem' (FMT; Morishima [38]). The FMT has generated an extensive literature (see, e.g., the seminal contributions by Okishio [40] and Morishima [38]). ${ }^{12}$ Nonetheless, the robustness

\footnotetext{
${ }^{12}$ For a counterpoint on the normative relevance of the FMT and an alternative explanation of the origins of profits,
} 
and theoretical relevance of the FMT have been put into question: according to critics, the FMT holds only under very restrictive assumptions concerning preferences, endowments, and technology and, in any case, the relation between exploitation and profits is spurious, as the FMT simply captures the productivity of the economy (for a comprehensive discussion, see Yoshihara [67]).

Yoshihara and Veneziani $[63,64]$ have recently shown that, contrary to the critics' claims, the main insights of the FMT continue to hold in static economies with general technologies and preferences. In this paper, we explicitly tackle the relation between exploitation, profits and growth, and provide a dynamic generalization of the FMT in a model with maximizing agents.

\section{The Model}

The economy consists of a sequence of non-overlapping generations. In each generation there is a set $\mathcal{N}_{c}=\left\{1, \ldots, N_{c}\right\}$ of capitalists with generic element $\nu$, and a set $\mathcal{N}_{w}=\left\{1, \ldots, N_{w}\right\}$ of workers with generic element $\eta$. Agents live for $T$ periods and are indexed by the date of birth $k T$, $k=0,1,2, \ldots, k T .{ }^{13}$ In every period $t$, they produce and exchange $n$ commodities and labor. Let $\left(p_{t}, w_{t}\right)$ denote the $1 \times(n+1)$ price vector in $t$, where $w_{t}$ is the nominal wage. ${ }^{14}$

We analyze the consequences of wealth inequalities in economies with incomplete capital markets, and model differences in behavior starkly. Let $\mathbf{0}$ be the null vector. In every $t$, each capitalist $\nu \in \mathcal{N}_{c}$ owns a $n \times 1$ vector of productive assets $\omega_{t}^{\nu} \geqq \mathbf{0},{ }^{15}$ where $\omega_{k T}^{\nu} \geq \mathbf{0}$ is the vector of endowments inherited, when born in $k T$. In every $t$, capitalists do not work but can hire workers in order to operate any activity of a standard Leontief technology $(A, L)$, where $A$ is a $n \times n$ non-negative, productive and indecomposable matrix of material input coefficients and $L>\mathbf{0}$ is a $1 \times n$ vector of direct labor coefficients. ${ }^{16}$ For every $\nu, y_{t}^{\nu}$ is the $n \times 1$ vector of activity levels that $\nu$ hires workers to operate at $t$.

Following Roemer [50, 52, 53], production takes time and capitalists must lay out in advance the capital necessary to finance production. However, agents cannot access credit markets to finance either production or accumulation. ${ }^{17}$ Formally, in every $t$ each capitalist $\nu$ has to use her wealth, $p_{t} \omega_{t}^{\nu}$, to obtain the necessary material inputs. At the end of the production period, capitalists use their net income to pay workers and to finance consumption and accumulation. Thus, for each $\nu$, in every $t, s_{t}^{\nu}$ is the $n \times 1$ vector of savings and $c_{t}^{\nu} \geqq \mathbf{0}$ is the $n \times 1$ consumption vector.

The choices available to workers are much more limited. Each worker $\eta \in \mathcal{N}_{w}$ possesses no physical capital, $\omega_{t}^{\eta}=\mathbf{0}$ in every $t$, but is endowed with one unit of labor. Therefore workers obtain income only by supplying labor and we assume that they use their income only to purchase consumption goods. ${ }^{18}$ To be precise, at all $t, z_{t}^{\eta}$ is $\eta$ 's labor supply and $c_{t}^{\eta}$ is $\eta$ 's $n \times 1$ consumption

\footnotetext{
see Hahnel [23].

${ }^{13}$ We focus on the empirically relevant case of economies with finite $T$. Both the model and our main conclusions, however, can be extended to the case with infinite $T$, albeit at the cost of an increase in notational intensity.

${ }^{14}$ Throughout the paper, all variables and vectors are assumed to belong to a Euclidean space $\mathbb{R}^{k}$ of appropriate dimensionality $k$.

${ }^{15}$ For all vectors $x, y \in \mathbb{R}^{p}, x \geqq y$ if and only if $x_{i} \geqq y_{i}(i=1, \ldots, p) ; x \geq y$ if and only if $x \geqq y$ and $x \neq y$; $x>y$ if and only if $x_{i}>y_{i}(i=1, \ldots, p)$.

${ }^{16}$ In the basic model, technology remains unchanged over time. We introduce technical progress in section 6 below. The other assumptions on technology can also be relaxed, albeit at the cost of a significant increase in technicalities (see, for example, the general convex cone economies analyzed in Veneziani and Yoshihara [65]).

${ }^{17}$ As in Patriarca and Vona [42] the economy displays intertemporal complementarities - whereby "production takes time and is carried on in vertical integrated firms with fixed proportions of capital and labor" (Patriarca and Vona [42], p.1643) - and markets open sequentially (produced inputs are bought before production takes place and must be financed out of beginning-of-period asset holdings).

${ }^{18}$ Our assumptions on saving behavior yield no significant loss of generality, either theoretically or formally. Theoretically, our aim is not to analyze social mobility, nor are we trying to explain earning inequalities arising from heterogeneous labor and skills. Formally, none of our key conclusions depends on ruling out workers' accumulation. In fact, the inclusion of workers' accumulation would strengthen our results on the transitory nature of inequalities in the basic economy without technical progress. Further, as shown below, along any equilibrium paths with accu-
} 
vector. Furthermore, the (work and consumption) choices available to workers are limited by the structural features of the economy and in particular by the presence of unemployment. Formally, for all $\eta \in \mathcal{N}_{w}$, in every $t$, there exists an upper bound $\widehat{z}_{t}^{\eta}$ to $\eta$ 's labor supply, which is determined by demand conditions.

We assume that, at all $t, c_{t}^{\nu} \geqq \mathbf{0}$ for all $\nu \in \mathcal{N}_{c}$, while there exists a reference consumption bundle $b>\mathbf{0}$, such that $c_{t}^{\eta} \geqq b$, for all $\eta \in \mathcal{N}_{w}$. Unlike capitalists, workers perform labor and need a minimum amount of consumption in return. ${ }^{19}$ This incorporates the idea that capitalists are not essential in production and, together with the assumption that $\omega_{t}^{\eta}=\mathbf{0}$, for all $\eta \in \mathcal{N}_{w}$ and all $t$, it starkly outlines differences between workers and capitalists. ${ }^{20}$

Although some aspects of behavior are determined by socio-economic status, we rule out heterogeneity in preferences over consumption. Formally, there is a continuous, strictly increasing, strictly quasi-concave, and homogeneous of degree one function $\phi: \mathbb{R}_{+}^{n} \rightarrow \mathbb{R}_{+}$, such that $\phi\left(c_{t}^{h}\right)$ describes agent $h$ 's welfare at $t$, where $h=\nu, \eta$, and we normalize $\phi$ by assuming that $\phi\left(c_{t}^{h}\right)=0$ whenever $c_{i t}^{h}=0$ for some good $i$.

Credit markets are incomplete and there is no intertemporal trade between agents. ${ }^{21}$ This is consistent with the lack of a pure accumulation motive - that is, the desire to maximize capital accumulation per se, which is often assumed in Marxist models (e.g., Morishima [37]; Roemer [51]). In our model, capitalists do not aim to maximize accumulation of capital per se, and production does not take place for production's own sake. However, Roemer's [50, 52, 53] static economies are generalized by allowing for intertemporal trade-offs during an agent's life.

Let $(p, w)=\left\{p_{t}, w_{t}\right\}_{t=k T, \ldots,(k+1) T-1}$ denote the path of the price vector during the lifetime of a generation. Let $y^{\nu}=\left\{y_{t}^{\nu}\right\}_{t=k T, \ldots,(k+1) T-1}$ denote $\nu$ 's lifetime plan of activity levels and let a similar notation hold for $c^{\nu}, s^{\nu}, \omega^{\nu}, z^{\eta}$, and $c^{\eta}$. As a shorthand notation, let "all $t$ " stand for "all $t, t=k T, \ldots,(k+1) T-1$." Let $0<\beta \leqq 1$ be the discount factor. Capitalist $\nu$ is assumed to choose $\xi^{\nu}=\left(y^{\nu}, c^{\nu}, s^{\nu}\right)$ to maximize lifetime welfare subject to the constraint that (1) net revenues (profits) are sufficient for consumption and savings, all $t$; (2) wealth is sufficient for production plans, all $t$; (3) the dynamics of assets is determined by savings, all $t$; and (4) $\nu$ 's descendants receive at least as many resources as she inherited. Formally, agent $\nu \in \mathcal{N}_{c}$ solves the following maximization program $\left(M P^{\nu}\right)$, whose value is denoted as $C\left(\omega_{k T}^{\nu}\right)$.

$$
M P^{\nu}: C\left(\omega_{k T}^{\nu}\right)=\max _{\xi^{\nu}} \sum_{t=k T}^{(k+1) T-1} \beta^{t} \phi\left(c_{t}^{\nu}\right),
$$

subject to

$$
\begin{aligned}
{\left[p_{t}(I-A)-w_{t} L\right] y_{t}^{\nu} } & \geqq p_{t} c_{t}^{\nu}+p_{t} s_{t}^{\nu}, \\
p_{t} A y_{t}^{\nu} & \leqq p_{t} \omega_{t}^{\nu}, \\
\omega_{t+1}^{\nu} & =\omega_{t}^{\nu}+s_{t}^{\nu}, \\
\omega_{(k+1) T}^{\nu} & \geqq \omega_{k T}^{\nu}, \\
y_{t}^{\nu} & \geqq \mathbf{0}, \omega_{t}^{\nu} \geqq \mathbf{0}, c_{t}^{\nu} \geqq \mathbf{0} .
\end{aligned}
$$

mulation, workers do not save, due to the downward pressure exercized by unemployment on the real wage. (This is reminiscent of a feature of the model by Banerjee and Newman ([5], p.291), whereby the abundance of labor drives the wage down which "makes it virtually impossible ... for workers to accumulate enough wealth".)

${ }^{19}$ The reference vector $b$ does not identify a physical subsistence bundle. Rather, we interpret it as a sociallydetermined basic consumption standard which must be reached in order for workers to supply labor in the capitalist sector (Banerjee and Newman [5]). We assume $b$ to be constant over time, but the model can be generalized to incorporate a time-varying $b_{t}$ reflecting evolving social norms, culture, and so on.

${ }^{20}$ In a less schematic model, if profits fall below some threshold, capitalists would start to work.

${ }^{21}$ As already noted, credit markets are also incomplete in the sense that, within each period, agents cannot activate production with external finance. As Roemer [50, 52, 53] has shown, however, this assumption yields no significant loss of generality. 
Similarly, worker $\eta \in \mathcal{N}_{w}$ chooses $\xi^{\eta}=\left(z^{\eta}, c^{\eta}\right)$ to maximize welfare subject to the constraint that at all $t$ : (5) revenues are sufficient for $\eta$ 's consumption; and (6) subsistence is reached. Furthermore, at all $t,(7)$ workers' labor supply is constrained both by their labor endowment and by labor market conditions, as captured by the (from the individual's viewpoint) exogenously given parameter $\widehat{z}_{t}^{\eta}$. Formally, agent $\eta \in \mathcal{N}_{w}$ solves the following maximization program $\left(M P^{\eta}\right)$.

$$
M P^{\eta}: \max _{\xi^{\eta}} \sum_{t=k T}^{(k+1) T-1} \beta^{t} \phi\left(c_{t}^{\eta}\right),
$$

subject to

$$
\begin{aligned}
w_{t} z_{t}^{\eta} & \geqq p_{t} c_{t}^{\eta}, \\
c_{t}^{\eta} & \geqq b, \\
\min \left[1, \widehat{z}_{t}^{\eta}\right] & \geqq z_{t}^{\eta} \geqq 0 .
\end{aligned}
$$

The optimization programs $M P^{\nu}$ and $M P^{\eta}$ allow us to investigate the relation between wealth inequalities, growth, and exploitation in a dynamic context. Given the absence of capital markets and of any explicit bequest motive, ${ }^{22}$ they are a natural generalization of Roemer's $[50,52,53]$ static profit or revenue maximization programs.

Let $\Omega_{k T}=\left(\omega_{k T}^{1}, \omega_{k T}^{2}, \ldots, \omega_{k T}^{N_{c}}\right)$. Let $E\left(\left(\mathcal{N}_{c}, \mathcal{N}_{w}\right),(A, L), \Omega_{k T},(\beta, \phi)\right)$, or as a shorthand notation $E_{k T}$, denote the economy with population $\left(\mathcal{N}_{c}, \mathcal{N}_{w}\right)$, technology $(A, L)$, endowments $\Omega_{k T}$, discount factor $\beta$ and welfare function $\phi$. At all $t$, let $y_{t}=\sum_{\nu \in \mathcal{N}_{c}} y_{t}^{\nu}, c_{t}^{c}=\sum_{\nu \in \mathcal{N}_{c}} c_{t}^{\nu}, \omega_{t}=\sum_{\nu \in \mathcal{N}_{c}} \omega_{t}^{\nu}$, $s_{t}=\sum_{\nu \in \mathcal{N}_{c}} s_{t}^{\nu}, c_{t}^{w}=\sum_{\eta \in \mathcal{N}_{w}} c_{t}^{\eta}$, and $z_{t}=\sum_{\eta \in \mathcal{N}_{w}} z_{t}^{\eta}$. Following Roemer [50,52, 53], the equilibrium concept can now be defined. ${ }^{23}$

Definition 1: A reproducible solution (RS) for $E_{k T}$ is a price vector $(p, w)$ and an associated set of actions $\left(\left(\xi^{\nu}\right)_{\nu \in \mathcal{N}_{c}},\left(\xi^{\eta}\right)_{\eta \in \mathcal{N}_{w}}\right)$ such that:

(i) $\xi^{\nu}$ solves $M P^{\nu}$ for all $\nu \in \mathcal{N}_{c}$;

(ii) $\xi^{\eta}$ solves $M P^{\eta}$ for all $\eta \in \mathcal{N}_{w}$;

(iii) $y_{t} \geqq A y_{t}+c_{t}^{c}+c_{t}^{w}+s_{t}$, for all $t$;

(iv) $A y_{t} \leqq \omega_{t}$, for all $t$;

(v) $L y_{t}=z_{t}$, for all $t$;

(vi) $\omega_{(k+1) T} \geqq \omega_{k T}$.

Conditions (i) and (ii) require agents to optimize given the individual and the aggregate constraints limiting their choices; (iii) and (iv) require that there be enough resources for consumption and saving plans, and for production plans, respectively, at all $t$; (v) states that the amount of labor performed in the economy must be sufficient for production plans at all $t$; (vi) requires that resources not be depleted by any given generation.

Definition 1 is an intertemporal extension of the concept of RS first defined by Roemer [50] and it provides a general notion of Marxian equilibrium (Veneziani and Yoshihara [65]). It is important to note that the concept of RS does not impose market clearing, and allows for an aggregate excess supply of produced goods, and, crucially, labor. ${ }^{24}$ Thus, Definition 1(v) is an ex post condition consistent with the existence of involuntary unemployment. For, although workers

\footnotetext{
${ }^{22}$ Though constraint 4 can also be interpreted as incorporating some sort of social norm on bequests.

${ }^{23}$ If technical change occurs as described in section 6 below, then the economy is more precisely described as $E\left(\left(\mathcal{N}_{c}, \mathcal{N}_{w}\right),\left(A, L_{k T}, \delta\right), \Omega_{k T},(\beta, \phi)\right)$ but everything else remains unchanged.

${ }^{24}$ This is consistent with the Classical economists' emphasis on the conditions for the "reproducibility" of the economic system, rather than market clearing. As Roemer ([50], p.507) put it, "The concern is with whether the economic system can reproduce itself: whether it can produce enough output to replenish the inputs used, and to reproduce the workers for another period of work."
} 
choose their labor supply optimally and aggregate labor supply equals labor demand ex post, labor market conditions act as a constraint on workers' choices ex ante in condition (7). In fact, by the monotonicity of $\phi$, in our framework, the standard labor market clearing condition at $t$ requires $L y_{t}=N_{w}$, whereas involuntary unemployment occurs at $t$ whenever $L y_{t}=z_{t}<N_{w}$.

Therefore, we say that a RS is unconstrained if $L y_{t}=z_{t}=N_{w}$, for all $t$, while a RS is constrained at $t^{\prime}$ if there exists some $t^{\prime}$ such that $N_{w}>z_{t^{\prime}}=L y_{t^{\prime}}$ and $w_{t^{\prime}} z_{t^{\prime}}=N_{w} p_{t^{\prime}}$. Because workers are identical, we assume that at a constrained RS, all of them work an equal amount of time which allows them to reach subsistence. Given the absence of a subsistence sector and of the public sector, this seems an appropriate way of capturing unemployment in this model. Moreover, we also focus on the minimal wage rate in the case of involuntary unemployment. ${ }^{25}$ Thus, formally, if a RS is constrained at $t^{\prime}$, then $\widehat{z}_{t^{\prime}}^{\eta}=\frac{L y_{t^{\prime}}}{N_{w}}$ and $c_{t^{\prime}}^{\eta}=b$, all $\eta \in \mathcal{N}_{w}$.

Given the focus on the persistence of exploitation and profits, the subset of RSs with stationary capital will be of particular interest. A stationary reproducible solution (SRS) for $E_{k T}$ is a RS such that, at all $t, c_{t}^{\nu}=c^{\nu}$ and $s_{t}^{\nu}=\mathbf{0}$, all $\nu \in \mathcal{N}_{c}$, and $c_{t}^{\eta}=c^{\eta}$, all $\eta \in \mathcal{N}_{w}$.

Definition 2 captures the idea of capital scarcity as requiring that the total supply of productive assets is limited, relative to current demand at a given RS $(p, w) .{ }^{26}$

Definition 2: Let $(p, w)$ be a RS for $E_{k T}$. The economy $E_{k T}$ is said to exhibit capital scarcity at $(p, w)$, in period $t$, if and only if $p_{t} A y_{t}^{\nu}=p_{t} \omega_{t}^{\nu}$, all $\nu \in \mathcal{N}_{c}$. If $p_{t} A y_{t}^{\nu}<p_{t} \omega_{t}^{\nu}$, some $\nu \in \mathcal{N}_{c}$, then capital is said to be abundant at $(p, w)$, in period $t$.

To be sure, Definition 2 is not the only possible way of defining capital scarcity. Yet it incorporates an intuitive and widespread notion of scarcity of productive factors that goes back, for example, to Solow's [60] classic analysis. For, as it will be shown below, by Definition 2, in the linear economies considered in this paper, the economy exhibits capital scarcity when aggregate capital is insufficient to guarantee the full employment of the labor force, i.e. when it is "capital limited" in Roemer's [52] sense. However, as in Solow's ([60], pp.83-84) analysis of linear economies, by Definition 2 an economy may also exhibit capital scarcity when aggregate endowments are such as to allow for the full employment of both capital and labor, and so, as Solow ([60], p.84) has argued, "both factors are scarce." 27

\section{Exploitation and profits}

We begin our analysis by deriving some preliminary results concerning the properties of RS's. Two properties immediately follow from the monotonicity of $\phi$. First, because at the solution to $M P^{\nu}$, $\omega_{(k+1) T}^{\nu}=\omega_{k T}^{\nu}$, all $\nu \in \mathcal{N}_{c}$, if $(p, w)$ is a RS for $E_{k T}$, then it is also a RS for $E_{(k+1) T}$. Hence, we can interpret $(p, w)$ as a steady state solution and focus on $E_{0}$ without loss of generality. Second, at any RS, it must be $p_{t}>\mathbf{0}$ and $w_{t}>0$, all $t$.

Then, it is immediate to show that at a RS for $E_{0}$, in every period, constraints (1) and (2) are binding for all capitalists.

Lemma 1: Let $(p, w)$ be a $R S$ for $E_{0}$. Then, for all $t$ :

(i) $\left[p_{t}(I-A)-w_{t} L\right] y_{t}^{\nu}=p_{t} c_{t}^{\nu}+p_{t} s_{t}^{\nu}$, all $\nu \in \mathcal{N}_{c}$;

(ii) if $p_{t} \geq p_{t} A+w_{t} L$, then $p_{t} A y_{t}^{\nu}=p_{t} \omega_{t}^{\nu}$, all $\nu \in \mathcal{N}_{c}$.

Let the profit rate of sector $i$ at $t$ be denoted as $\pi_{i t}=\frac{\left[p_{t}(I-A)-w_{t} L\right]_{i}}{p_{t} A_{i}}$. The next Lemma proves that at a RS, in every period profits are non-negative and profit rates are equalized across sectors.

\footnotetext{
${ }^{25}$ Note that at a RS, if $N_{w}>z_{t^{\prime}}=L y_{t^{\prime}}$ for some $t^{\prime}$ then this does not necessarily imply $w_{t^{\prime}} z_{t^{\prime}}=N_{w} p_{t^{\prime}} b$. If $w_{t^{\prime}} z_{t^{\prime}}>N_{w} p_{t^{\prime}} b$, then $c_{t^{\prime}}^{\eta} \geq b$ holds for all $\eta \in \mathcal{N}_{w}$.

${ }^{26}$ To be precise, a RS should be denoted as $\left(p, w,\left(\xi^{\nu}\right)_{\nu \in \mathcal{N}_{c}},\left(\xi^{\eta}\right)_{\eta \in \mathcal{N}_{w}}\right)$. We write $(p, w)$ for notational simplicity.

${ }^{27}$ Thus, capital scarcity as defined in Definition 2 encompasses Roemer's [52] concept of a "capital limited" economy but generalizes it. We are grateful to an anonymous referee for pointing out the latter concept.
} 
Lemma 2: Let $(p, w)$ be a $R S$ for $E_{0}$. Then, at all $t, \pi_{i t} \geqq 0$, for at least some $i$. Furthermore, if either $\pi_{i t}>0$, some $i$, or $c_{t}^{c}+c_{t}^{w}+s_{t}>\mathbf{0}$, then $\pi_{i t}=\pi_{t}$, all $i$.

Proof. 1. Suppose that there is some $t$ such that $p_{i t}<p_{t} A_{i}+w_{t} L_{i}$, all $i$. Then $y_{i t}^{\nu}=0$, all $i$, $\nu \in \mathcal{N}_{c}$, for all $\xi^{\nu}$ that solve $M P^{\nu}$, and thus $y_{t}=0$. By Definition $1(\mathrm{v})$, this implies $z_{t}^{\eta}=0$, all $\eta \in \mathcal{N}_{w}$, which violates Definition 1(ii).

2. Suppose that there is some $t$ such that either $\pi_{i t}>0$, some $i$, or $c_{t}^{c}+c_{t}^{w}+s_{t}>\mathbf{0}$, but $\pi_{l t}<\pi_{j t}$, some $j, l$. Because wealth is used only to activate maximum profit rate processes, it follows that for all $\nu \in \mathcal{N}_{c}, y_{l t}^{\nu}=0$, for all $\xi^{\nu}$ that solve $M P^{\nu}$, and thus $y_{l t}=0$. However, under the hypotheses stated, $A_{l} y_{t}+c_{l t}^{c}+c_{l t}^{w}+s_{l t}>\mathbf{0}$, which contradicts Definition 1(iii).

By Lemma 2, at a SRS, $\pi_{i t}=\pi_{t}$, all $i$ and all $t$. More generally, at any RS such that $\pi_{i t}=\pi_{t}$, all $i$, we can consider price vectors such that $p_{t}=\left(1+\pi_{t}\right) p_{t} A+w_{t} L$, all $t$. Furthermore, labor can be chosen as the numéraire, setting $w_{t}=1$, all $t$, and in what follows we focus on RS's of the form $(p, \mathbf{1})$, where $\mathbf{1}=(1, \ldots, 1)^{\prime}$.

Let $\lambda=L(I-A)^{-1}$ be the $1 \times n$ vector of Leontief employment multipliers, measuring the amount of labor (directly and indirectly) necessary to produce goods. By the assumptions on $A$, $\lambda>\mathbf{0}$ and throughout the paper we shall assume that the economy is sufficiently productive to guarantee the reproduction of workers and be able to grow. Formally, $\lambda b<1$.

Let $y=\sum_{t=0}^{T-1} y_{t}$ and $c^{w}=\sum_{t=0}^{T-1} c_{t}^{w}$. Generalizing Roemer [51,52], the amount of labor received by workers at $t$ is defined as the amount of labor embodied, or contained, in their consumption, $\lambda c_{t}^{w}$. Similarly, the amount of labor received by workers during their whole life is defined as $\lambda c^{w}$. Then, Roemer's definition of UE exploitation can be extended to the intertemporal context. ${ }^{28}$

Definition 3: The within-period (WP) exploitation rate at $t$ is $e_{t}=\frac{\left(L y_{t}-\lambda c_{t}^{w}\right)}{\lambda c_{t}^{w}}$ and the whole-life (WL) exploitation rate is $e=\frac{\left(L y-\lambda c^{w}\right)}{\lambda c^{w}}$.

Both definitions convey potentially normatively relevant information and provide important insights on the foundations of inequalities in capitalist economies. Nonetheless, below we shall focus on the WP definition because it is more appropriate in the analysis of the relation between inequality and growth, and of the evolution of exploitation over time. ${ }^{29}$

Theorem 1 proves the existence of a robust correspondence between UE exploitation and the functional distribution of income. To discuss it, we introduce a reasonable restriction on the welfare function $\phi .^{30}$

Assumption 1 (A1): All commodities are normal goods, and are gross complements to each other.

Then, we have the following characterization. ${ }^{31}$

Theorem 1 Let $(p, \mathbf{1})$ be a $R S$ for $E_{0}$ with $\pi_{i t}=\pi_{t}$, all $i$ and all $t$. Then (i) at all $t, e_{t}>0$ if and only if $\pi_{t}>0$. Furthermore, (ii) $e>0$ if and only if $\pi_{t}>0$, some $t$. Finally, (iii) if the economy $E_{0}$ satisfies A1, then at each $t$, there is a monotone-increasing functional relationship $\pi\left(e_{t}\right)=\pi_{t}$.

\footnotetext{
${ }^{28}$ With technical change, the definition of the WL exploitation rate would need to be adjusted accordingly.

${ }^{29}$ As argued in Veneziani [62], the WP approach is also more suitable to capture the structural features of exploitative relations. It is worth reiterating, however, that a focus on the WP definition does not mean that WL exploitation is normatively irrelevant. In our economy, the existence of WP exploitation implies WL exploitation, and the disappearance of WP exploitation after a certain period does not make economic relations normatively unobjectionable. We are grateful to an anonymous referee for raising this issue.

${ }^{30} \mathrm{An}$ example of welfare function satisfying A1 is the Cobb-Douglas function.

${ }^{31}$ The proofs of all theorems are in Appendix A.
} 
Theorem 1 proves that a correspondence exists between positive profits and the existence of UE exploitation in general dynamic economies with intertemporally optimizing agents. From a normative perspective, Theorem 1 is important because it establishes a link between wealth inequalities, the functional distribution of income, and exploitation: given private ownership of productive assets, profits are a counterpart of the transfer of social surplus and social labor from asset-poor agents to the wealthy. Equilibria in which a higher portion of social labor is transferred onto capitalists are also characterized by a functional distribution of income that is more favorable to them, and vice versa.

However, because the rate of profit is one of the key determinants of investment decisions, and of the long-run dynamics of capitalist economies, Theorem 1 can also be interpreted as providing a link between exploitation and growth. In equilibrium, higher exploitation rates imply higher profit rates which in turn imply more funds for capitalist accumulation. Further, by Theorem 1, it is possible to show that there is no RS with persistent accumulation and persistent exploitation.

In fact, if $e_{t}>0$, all $t$, then by Theorem 1 and Lemma 1(ii), and noting that $p_{t}>\mathbf{0}$, at a RS $L y_{t}=L A^{-1} \omega_{t}$, all $t$. By Definition 1(ii) and (v), it must therefore be $L A^{-1} \omega_{t} \leqq N_{w}$, all $t$. Hence, if $\omega_{t+1}>\omega_{t}$, all $t, T-1>t \geqq 0$, then $L A^{-1} \omega_{t}<N_{w}$, and the RS is constrained at all $t$, $T-1>t \geqq 0$. Therefore $c_{t}^{\eta}=b$, all $\eta$, and $p_{t} b=\frac{L A^{-1} \omega_{t}}{N_{w}}$, all $t, T-1>t \geqq 0$. By Lemma $1(\mathrm{i})$, and noting that $p_{t}>\mathbf{0}$, at a RS $(I-A) y_{t}=s_{t}+c_{t}^{c}+c_{t}^{w}$, all $t$, which implies $s_{t}=(I-A) A^{-1} \omega_{t}-c_{t}^{c}-c_{t}^{w}$, all $t$, or by the previous arguments, $\omega_{t+1}=A^{-1} \omega_{t}-c_{t}^{c}-N_{w} b$, all $t, T-1>t \geqq 0$.

Given the linearity of $M P^{\nu}$, there is at most one period in which, for any $\nu \in \mathcal{N}_{c}$, at the solution to $M P^{\nu}$, both savings and consumption are positive at a constrained RS with accumulation. ${ }^{32}$ Hence, given that capitalists are identical there is a period $\tau$ such that $c_{t}^{c}=\mathbf{0}$, all $t \geqq \tau$, and $\omega_{t+1}=A^{-1} \omega_{t}-N_{w} b$, all $t \geqq \tau$, which implies $\omega_{t}=\left(A^{-1}\right)^{t-\tau}\left[\omega_{\tau}-\omega_{S}\right]+\omega_{S}$, all $t \geqq \tau$, where $\omega_{S}=N_{w} A(I-A)^{-1} b$. Thus, by the productivity of $A$, given that workers' subsistence requires $\omega_{t} \geqq \omega_{S}$, all $t$, if $T$ is sufficiently big, labor demand exceeds supply after a finite number of periods, driving $\pi_{t}$ and $e_{t}$ to zero. This can be summarized as follows.

Proposition 1: For all $T>0$, there is a $T^{\prime}>T$ such that there is no $R S$ with $\omega_{t+1}>\omega_{t}$, all $t$, $T^{\prime}-1>t \geqq 0$, and $e_{t}>0$, all $t$.

In other words, persistent accumulation and persistent exploitation and profits are inconsistent. At a broad conceptual level, this conclusion echoes a classic argument by Kalecki [25], according to which capitalists will tend to have negative attitudes towards policies that promote growth and full employment. For Proposition 1 suggests that, absent any countervailing factors, capitalists will be collectively concerned with any long-run sustained accumulation that may affect capital scarcity, even though individually they may regard growth paths favorably.

The intuition behind Proposition 1 is simple. In our linear economy, if capitalists had a persistent incentive to accumulate, this would yield a continual increase in the demand for labor which, provided the time horizon is sufficiently long, would eventually exceed the fixed labor supply making capital abundant and leading profits and exploitation to zero. It is important to note, however, that Proposition 1 does not prove that there are growth paths in which profits and exploitation disappear in equilibrium, because the anticipated fall in the profit rate might discourage rational agents from investing.

In the next sections, we explore optimal behavior and the equilibrium dynamics of the economy.

\section{Inequalities, Exploitation, and Time Preference}

This section analyzes the dynamic foundations of exploitative relations, focusing on stationary reproducible solutions. This is due to the theoretical relevance of SRS's, as discussed in Veneziani

\footnotetext{
${ }^{32}$ This is proved rigorously below; see e.g. the analysis of $M P^{\nu}$ in the proof of Theorem 4 .
} 
[62], but also because they represent a benchmark solution whereby the labor market may clear at all $t$. Lemma 3 provides a necessary condition for the existence of a SRS.

Lemma 3: Let $(p, \mathbf{1})$ be a SRS for $E_{0}$ with $\pi_{t}>0$, all $t$. Then $\beta\left(1+\pi_{t+1}\right)=1$, all $t$.

Proof. 1. For all $\nu \in \mathcal{N}_{c}$, by Lemma 1, at any RS with $\pi_{t}>0$, all $t$, it must be $p_{t} c_{t}^{\nu}=\pi_{t} p_{t} \omega_{t}^{\nu}-p_{t} s_{t}^{\nu}$, all $t$. At a SRS, the latter expression becomes $p_{t} c^{\nu}=\pi_{t} p_{t} \omega_{0}^{\nu}$, all $t, \nu$, which implies $c^{\nu} \geq \mathbf{0}$.

2. Suppose, by way of contradiction, that $\beta\left(1+\pi_{t^{\prime}+1}\right)>1$, some $t^{\prime}<T-1$. Take any capitalist $\nu \in \mathcal{N}_{c}$. Consider a one-period perturbation of $\nu^{\prime}$ 's optimal choice such that $p_{t^{\prime}} \mathrm{d} c_{t^{\prime}}^{\nu}=-p_{t^{\prime}} \mathrm{d} s_{t^{\prime}}^{\nu}$, $p_{t^{\prime}+1} \mathrm{~d} c_{t^{\prime}+1}^{\nu}=\pi_{t^{\prime}+1} p_{t^{\prime}+1} \mathrm{~d} \omega_{t^{\prime}+1}^{\nu}-p_{t^{\prime}+1} \mathrm{~d} s_{t^{\prime}+1}^{\nu}, \mathrm{d} \omega_{t^{\prime}+1}^{\nu}=\mathrm{d} s_{t^{\prime}}^{\nu}=-\mathrm{d} s_{t^{\prime}+1}^{\nu}$.

3. Since $\phi$ is homothetic, $c_{t}^{\nu}=c^{\nu}$ implies that at a SRS, at all $t$ it must be $p_{t+1}=k_{t} p_{t}$ for some $k_{t}>0$. Therefore consider $\mathrm{d} c_{t^{\prime}}^{\nu}=h_{t^{\prime}} c^{\nu}$ and $\mathrm{d} c_{t^{\prime}+1}^{\nu}=h_{t^{\prime}+1} c^{\nu}$ for some $h_{t^{\prime}}, h_{t^{\prime}+1} \geq 0$, and the one period perturbation can be written as $h_{t^{\prime}} p_{t^{\prime}} c^{\nu}=-p_{t^{\prime}} \mathrm{d} s_{t^{\prime}}^{\nu}$ and $h_{t^{\prime}+1} p_{t^{\prime}} c^{\nu}=\pi_{t^{\prime}+1} p_{t^{\prime}} \mathrm{d} s_{t^{\prime}}^{\nu}+p_{t^{\prime}} \mathrm{d} s_{t^{\prime}}^{\nu}$.

4. By the homogeneity of $\phi$ it follows that $\phi\left(c^{\nu}+\mathrm{d} c_{t^{\prime}}^{\nu}\right)+\beta \phi\left(c^{\nu}+\mathrm{d} c_{t^{\prime}+1}^{\nu}\right)=\left(1+h_{t^{\prime}}\right) \phi\left(c^{\nu}\right)+$ $\left(1+h_{t^{\prime}+1}\right) \beta \phi\left(c^{\nu}\right)>\phi\left(c^{\nu}\right)+\beta \phi\left(c^{\nu}\right)$ if and only if $h_{t^{\prime}}+h_{t^{\prime}+1} \beta=\left[-1+\beta\left(1+\pi_{t^{\prime}+1}\right)\right] \frac{p_{t^{\prime}}}{p_{t^{\prime}} c^{\nu}} \mathrm{d} s_{t^{\prime}}^{\nu}>$ 0 . Therefore, if $\beta\left(1+\pi_{t^{\prime}+1}\right)>1$, there is a sufficiently small $\mathrm{d} s_{t^{\prime}}^{\nu}$ with $p_{t^{\prime}} \mathrm{d} s_{t^{\prime}}^{\nu}>0$ such that $h_{t^{\prime}}+h_{t^{\prime}+1} \beta>0$, a contradiction. A similar argument holds if $\beta\left(1+\pi_{t^{\prime}+1}\right)<1$, some $t^{\prime}<T-1$.

Intuitively, if $\beta\left(1+\pi_{t^{\prime}+1}\right)>1$, some $t^{\prime}$, then the cost (in terms of overall welfare) of reducing consumption at $t^{\prime}$ is lower than the benefit of saving, producing and consuming in $t^{\prime}+1$, and vice versa if $\beta\left(1+\pi_{t^{\prime}+1}\right)<1$. Only if $\beta\left(1+\pi_{t^{\prime}+1}\right)=1$ are costs and benefits equal.

Let $\frac{1}{1+\pi}$ be the Frobenius eigenvalue of $A$ : by the assumptions on $A, \widetilde{\pi}>0$. Let $\pi_{\beta} \equiv \frac{1-\beta}{\beta}$ and let $p_{\beta}$ denote the solution of $p=\left(1+\pi_{\beta}\right) p A+L$ : for all $\pi_{\beta} \in[0, \tilde{\pi}), p_{\beta}$ is well defined and strictly positive. By the homotheticity of $\phi$, let $c_{\beta}$ denote a vector identifying the optimal proportions of the different consumption goods corresponding to $p_{\beta} \cdot{ }^{33}$ Theorem 2 analyzes $M P^{\nu} \cdot{ }^{34}$

Theorem 2 (i) Let $1>\beta>\frac{1}{1+\widetilde{\pi}}$. If $\pi_{t}=\pi_{\beta}$, all $t$, then for all $\nu \in \mathcal{N}_{c}$ there is an optimal $\xi^{\nu}$ such that $s_{t}^{\nu}=\mathbf{0}$, all $t$, and $C\left(\omega_{0}^{\nu}\right)=\phi\left(c_{\beta}\right)\left(1-\beta^{T}\right) \frac{p_{\beta} \omega_{0}^{\nu}}{\beta p_{\beta} c_{\beta}}$. (ii) Let $\beta \leqq 1$. If $\pi_{t}=0$, all $t$, then for all $\nu \in \mathcal{N}_{c}$ there is an optimal $\xi^{\nu}$ such that $s_{t}^{\nu}=\mathbf{0}$, all $t$, and $C\left(\omega_{0}^{\nu}\right)=0$.

Consider a subset of the set of conceivable aggregate endowments of productive assets, namely vectors of the form $\omega_{0}=\gamma_{0} N_{w} A(I-A)^{-1} b$, where $\gamma_{0}$ is a positive real number capturing the abundance of aggregate capital relative to the subsistence requirements of workers. If $\gamma_{0}=1$, then aggregate endowments are barely sufficient to guarantee the subsistence of all workers and it is not difficult to show that the only RS for $E_{0}$ requires $\pi_{t}=0$ and $s_{t}=\mathbf{0}$, all $t$.

Let $\pi^{\prime}$ be defined by $\gamma_{0} \lambda b=L\left[I-\left(1+\pi^{\prime}\right) A\right]^{-1} b: \pi^{\prime}$ is the profit rate such that a worker supplying an amount of labor $\gamma_{0} \lambda b$ can purchase the subsistence bundle $b$ at prices $p^{\prime}=L\left[I-\left(1+\pi^{\prime}\right) A\right]^{-1}$.

Given Theorem 2, the next result proves the existence of a SRS. ${ }^{35}$

Theorem 3 Let $\omega_{0}=\gamma_{0} N_{w} A(I-A)^{-1} b, \gamma_{0}>1$. Let $\pi^{\prime}$ be defined by $\gamma_{0} \lambda b=L\left[I-\left(1+\pi^{\prime}\right) A\right]^{-1} b$.

(i) Let $\gamma_{0} \lambda b<1$. If $\beta\left(1+\pi^{\prime}\right)=1$ and $c_{\beta}=k b$ for some $k>0$, there is a SRS for $E_{0}$ with $\pi_{t}=\pi^{\prime}$, all $t$

(ii) Let $\gamma_{0} \lambda b=1$. Let $\beta \in\left[\frac{1}{\left(1+\pi^{\prime}\right)}, 1\right)$ be such that $c_{\beta}=k b$ for some $k>0$. Then there is a SRS for $E_{0}$ with $\pi_{t}=\pi_{\beta}$, all $t$;

(iii) Let $\gamma_{0} \lambda b \leqq 1$. If $\beta=1$, there is a SRS for $E_{0}$ such that at all $t, \pi_{t}=0$ and $p_{t} A y_{t}^{\nu}=p_{t} \omega_{t}^{\nu}$, all $\nu \in \mathcal{N}_{c}$. Further, there is no SRS with $\pi_{t}>0$, some $t$.

\footnotetext{
${ }^{33}$ The vector $c_{\beta}$ is determined up to a scalar transformation. If $\phi_{i}^{\prime}$ denotes the partial derivative of $\phi$ with respect to the $i$-th entry, then $\frac{\phi_{i}^{\prime}\left(c_{\beta}\right)}{\phi_{j}^{\prime}\left(c_{\beta}\right)}=\frac{p_{i \beta}}{p_{j \beta}}$, for all $i, j$.

${ }^{34}$ In the case with $\pi_{t}=0$, all $t$, Theorem 2 does not rule out the possibility that for some $\nu \in \mathcal{N}_{c}, s_{t}^{\nu} \neq \mathbf{0}$, for some $t$, at the solution to $M P^{\nu}$. However, for all $\nu \in \mathcal{N}_{c}$ at any $\xi^{\nu}$ that solves $M P^{\nu}$, it must be $\lambda s_{t}^{\nu}=0$, all $t$.

${ }^{35}$ The restriction $\omega_{0}=\gamma_{0} N_{w} A(I-A)^{-1} b$ is necessary given the linearity of $M P^{\nu}$ and $M P^{\eta}$. No theoretical conclusion depends on this restriction, which in any case encompasses a rather large set of economies.
} 
Theorem 3 significantly strengthens and extends the results in Veneziani [62]. Consider economies in which aggregate initial assets are above the minimum barely sufficient to guarantee workers' subsistence $\left(\gamma_{0}>1\right)$, but below the level that would make aggregate labor demand higher than aggregate labor supply $\left(\gamma_{0} \lambda b \leqq 1\right)$. On the one hand, Theorem 3(i)-(ii) state that the dynamic economy with maximizing agents displays persistent exploitation - and possibly persistent unemployment, - if profits are consumed at all $t$ and capitalists discount the future. ${ }^{36}$ On the other hand, however, this result crucially depends on a strictly positive rate of time preference (Theorem 3(iii)): if capitalists do not discount the future, then there exists no stationary equilibrium with positive profits. There exists, instead, a stationary equilibrium with no profit (and no exploitation) in which capital is scarce in the sense of Definition 2. Further, if $\gamma_{0} \lambda b=1$, the magnitude of inequalities and exploitation will also depend on $\beta .^{37}$

This suggests that Roemer's [52, 53] claim that a differential distribution of (scarce) property and competitive markets are sufficient institutions to generate persistent UE exploitation may need to be at least qualified, and it is unclear that UE exploitation can be reduced to a focus on asset inequalities. For Theorem 3 proves that, absent time preference, exploitation is not a persistent feature of a competitive economy at a stationary RS, even if wealth inequalities endure and capital remains scarce in the sense of Definition 2. An exclusive focus on the differential ownership of (scarce) productive assets seems therefore insufficient to explain the origins of persistent UE exploitation, and asset inequalities alone are not all that matters when evaluating capitalist economies from a normative perspective. Something else is indispensable to generate persistent UE exploitation, which is therefore normatively as important as asset inequalities themselves.

In the next section, we explore further the foundations of persistent UE exploitation, going beyond the stationary equilibria considered in Theorem 3, and examine the relation between growth and inequalities in the distribution of income, and in the exchange of labor.

\section{Stable Growth and Distribution}

In this section, in order to focus on the key theoretical issues and on macrodynamics, we consider a special case of the $n$-good economies analyzed thus far by setting $n=1$. The model and notation remain the same, with obvious adaptations and letting $\phi$ be the identity function. ${ }^{38}$ Further, we restrict our attention to the empirically relevant case of economies in which $T$ can be arbitrarily large but remains finite.

Sections 4-5 suggest that asset inequalities (and competitive markets) alone cannot fully explain exploitative relations in dynamic capitalist economies. Persistent growth and exploitation are inconsistent and even if the economy does not grow, persistent exploitation is possible only if $\beta<1$. This section explores further the relation between exploitation, time preference, and growth, by focusing on stable growth paths in which the economy grows for a certain number of periods and eventually reaches a steady state. ${ }^{39}$

\footnotetext{
${ }^{36}$ Observe that the proof of existence in cases (i) and (ii) of the theorem are premised on the condition that $c_{\beta}=k b$ for some $k>0$. This condition on the agents' optimal consumption bundle is due to the linearity of the model and the presence of a subsistence constraint. None of our conclusions depend on this restriction, and the existence of a SRS can be proved under more general assumptions although at a cost of a significant increase in technicalities.

${ }^{37}$ Theorems 2-3 also characterize inter-capitalist inequalities as a different phenomenon from exploitation. In fact, at a SRS with $\pi_{t}=\frac{1-\beta}{\beta}>0$, all $t$, by Theorem 2 for any two capitalists $\nu$ and $\mu, C\left(\omega_{0}^{\nu}\right)>C\left(\omega_{0}^{\mu}\right)$ if and only if $p_{\beta} \omega_{0}^{\nu}>p_{\beta} \omega_{0}^{\mu}$. Instead, if $\pi_{t}=0$, all $t$, then $C\left(\omega_{0}^{\nu}\right)=0$, all $\nu$.

${ }^{38}$ The main conclusions of this section can be extended to $n$-good economies, albeit at the cost of a significant increase in technicalities. The key definitions and propositions are formulated so as to suggest the relevant $n$-good extensions.

${ }^{39}$ The notion of stability here does not refer to the concept of asymptotic stability in dynamical systems. The growth paths in Definition 4 are stable in the sense that the economy eventually reaches a stationary state. Observe
} 
Definition 4: A stable growth path (SGP) for $E_{0}$ is a RS such that there is a period $t^{\prime}>0$ such that $\omega_{t+1}=\left(1+g_{t}\right) \omega_{t}, g_{t}>0$, for all $t<t^{\prime}$, and $\omega_{t+1}=\omega_{t}$, all $t, T-1>t \geqq t^{\prime}$.

For all $t$, let $\omega_{t}=\gamma_{t} N_{w} A(1-A)^{-1} b$, so that any conditions on aggregate capital $\omega_{t}$ can be equivalently expressed as conditions on $\gamma_{t}$. Lemma 4 confirms the relevance of SRS's as a theoretical benchmark: only at a SRS can equilibrium in the labor market and exploitation exist at all $t$.

Lemma 4: If $(p, \mathbf{1})$ is an unconstrained RS for $E_{0}$ such that the economy exhibits capital scarcity at $t$, then $\gamma_{t} \lambda b=1$.

Proof. At a RS with capital scarcity at $t$, it must be $y_{t}=A^{-1} \omega_{t}$. Therefore, $L y_{t}=\gamma_{t} N_{w} \lambda b$, and since the RS is unconstrained, $L y_{t}=z_{t}=N_{w}$, which holds if and only if $\gamma_{t} \lambda b=1$.

In general, if a RS is unconstrained from $t^{\prime}$ onwards, then $\gamma_{t} \lambda b=1$, all $t \geqq t^{\prime}$, and thus SRS's are a natural benchmark for all accumulation paths with persistent capital scarcity, which lead to a stationary state with equilibrium in the labor market. Instead, if $\gamma_{t} \lambda b<1$, the economy is constrained at $t$. Proposition 2 rules out paths where capital becomes abundant.

Proposition 2: Let $\omega_{0}=\gamma_{0} N_{w} A(1-A)^{-1} b, \gamma_{0}>1$, and $\gamma_{0} \lambda b \leqq 1$. Suppose $\beta<1$. Then there is no RS such that there exists a period $\widehat{t}$ such that the economy exhibits capital scarcity at all $t \leqq \widehat{t}$ but $L A^{-1} \omega_{\widehat{t}+1}>N_{w}$.

Proof: 1 . Suppose that there is a RS such that $L A^{-1} \omega_{\widehat{t}} \leqq N_{w}$ but $L A^{-1} \omega_{\widehat{t}+1}>N_{w}$, some $\widehat{t}$. Then $\pi_{\widehat{t}}>0$ but $\pi_{\widehat{t}+1}=0$ since capital is abundant at $\widehat{t}+1$.

2. For all $\nu \in \mathcal{N}_{c}, c_{\hat{t}}^{\nu}=\pi_{\hat{t}} \omega_{\widehat{t}}^{\nu}-s_{\widehat{t}}^{\nu}$ and $c_{\hat{t}+1}^{\nu}=-s_{\hat{t}+1}^{\nu} \geqq 0$. If $s_{\widehat{t}+1}^{\nu}<0$, some $\nu \in \mathcal{N}_{c}$, then since $\beta\left(1+\pi_{\widehat{t}+1}\right)<1$, there is a feasible perturbation of the savings path with $\mathrm{d} s_{\widehat{t}}^{\nu}=-\mathrm{d} s_{\widehat{t}+1}^{\nu}<0$, which increases $\nu$ 's welfare, contradicting optimality.

3. Let $s_{\widehat{t}+1}^{\nu}=0$, all $\nu \in \mathcal{N}_{c}$. Since $s_{\widehat{t}+1}=0$ then $\omega_{\widehat{t}+2}=\omega_{\widehat{t}+1}$, so that $\pi_{\widehat{t}+2}=0$ and $\beta\left(1+\pi_{\hat{t}+2}\right)<1$. Again, for all $\nu \in \mathcal{N}_{c}, s_{\hat{t}+2}^{\nu}<0$ cannot be optimal. Therefore $s_{\widehat{t}+2}^{\nu}=0$, all $\nu \in \mathcal{N}_{c}$, and $\pi_{\widehat{t}+3}=0$; and so on.

4. By construction, $\omega_{\widehat{t}+1}>\omega_{0}$. Hence, individual optimality implies $\sum_{l=\widehat{t}+1}^{T-1} s_{l}^{\nu}<0$, all $\nu \in \mathcal{N}_{c}$, which contradicts $s_{l}^{\nu}=0$, for all $\nu \in \mathcal{N}_{c}$ and all $T-1 \geqq l \geqq \widehat{t}+1$.

Proposition 2 shows that overaccumulation is not an equilibrium because the fall of the profit rate to zero would rather lead capitalists to anticipate consumption, if $\beta<1$. Thus, it confirms the importance of time preference for the persistence of exploitation in Roemer's theory: if $\beta=1$, overaccumulation and profits falling to zero are not ruled out.

Given Proposition 2, Theorem 4 characterizes stable growth paths.

Theorem 4 Let $\omega_{0}=\gamma_{0} N_{w} A(1-A)^{-1} b$ and $\gamma_{0}>1$. Let $(p, \mathbf{1})$ be a SGP for $E_{0}$ such that $\gamma_{t} \lambda b \leqq 1$, all t. At all $t$, define $g_{t}^{\prime}=\frac{\left[\left(\gamma_{t}-1\right) N_{w} b-c_{t}^{c}\right] \tilde{\pi}}{\gamma_{t} N_{w} b}$. Then:

(i) $\omega_{t+1}=\left(1+g_{t}^{\prime}\right) \omega_{t}$, all $t<t^{\prime}$, and $p_{t+1}=\left(1+g_{t}^{\prime}\right) p_{t}$, all $t<t^{\prime}-1$. Furthermore, if $\beta<1$ then $g_{t}^{\prime}=\pi_{t}$, all $0<t<t^{\prime}-1$, while if $\beta=1$ then $g_{t}^{\prime}=\pi_{t}$, all $t<t^{\prime}-1$.

(ii) If $\beta<1$ and $\pi_{t}>0$, all $t, T-2 \geqq t \geqq t^{\prime}$, then $\beta\left(1+\pi_{t+1}\right)=1$, all $t, T-2 \geqq t \geqq t^{\prime}$. If $\beta=1$, there is no $t, T-2 \geqq t \geqq t^{\prime}$, such that $\pi_{t}>0$ and $\pi_{t+j}>0$, some $j>0$.

In other words, at any SGP, prior to $t^{\prime}$, the economy accumulates at the maximal rate, which coincides with the profit rate, and commodity prices grow at the same pace. As $p_{t}$ and the profit rate increase, the growth rate of the economy is not constant over time. Except for period $t=0$ the behavior of the economy in the first phase is the same regardless of whether agents discount

that if $T=2$, then at any SGP the condition in the second part of Definition 4 is vacuously satisfied. 
the future or not (Theorem 4(i)). After $t^{\prime}$, the economy is stationary: if $\beta<1$ then the profit rate is positive and equal to the value identified in Theorem 3(ii). If $\beta=1$ then the only profit rate consistent with a stationary path of capital after $t^{\prime}$ is $\pi_{t}=0$ (Theorem 4(ii)).

At a general level, Theorem 4 provides a clear link between the growth rate and the profit rate: profits are the engine of growth and to the extent that profitability, and the functional distribution of income, are related to income inequalities, Theorem 4 suggests that inequalities are indeed a necessary condition for economic growth in capitalist economies. ${ }^{40}$ Further, as in Classical models, Theorem 4 establishes a negative relation between capitalist consumption and growth (for a given workers' subsistence bundle $b$ ), and $g_{t}^{\prime}$ can be shown to coincide with the growth rate of von Neumann-Sraffa models (e.g., von Neumann [66]; Kurz and Salvadori [30] p.102ff).

The next result characterizes capitalists' optimal saving paths with accumulation.

Theorem 5 Let $(p, \mathbf{1})$ be such that $\pi_{t}>\pi_{\beta}$, all $t \leqq \tau$, and $\pi_{t}=\pi_{\beta}$, all $T-1 \geqq t \geqq \tau+1$, for some $\tau, T-1 \geqq \tau \geqq 0$. Then, for all $\nu \in \mathcal{N}_{c}$ : (i) $\omega_{t+1}^{\nu}=\left(1+\pi_{t}\right) \omega_{t}^{\nu}$, all $t \leqq \tau-1$, $\omega_{t+1}^{\nu}=\left(1+g_{t}\right) \omega_{t}^{\nu}$, all $g_{t} \in\left[0, \pi_{\beta}\right]$, all $t, T-2 \geqq t \geqq \tau$, and $\omega_{T}^{\nu}=\omega_{0}^{\nu}$, is optimal, and (ii) $C\left(\omega_{0}^{\nu}\right)=\left[\beta^{\tau} \Pi_{i=0}^{\tau}\left(1+\pi_{i}\right)-\beta^{T-1}\right] \omega_{0}^{\nu}$.

Let $\pi^{\prime}$ be defined by $1=L\left[1-\left(1+\pi^{\prime}\right) A\right]^{-1} b: \pi^{\prime}$ is the profit rate such that a worker supplying one unit of labor would be able to purchase the subsistence bundle $b$ at prices $p^{\prime}=L\left[1-\left(1+\pi^{\prime}\right) A\right]^{-1}$.

Recall that by definition the maximum profit rate that can be obtained is given by $\tilde{\pi}=\frac{1-A}{A}$. Let the sequence $\left\{\bar{\gamma}_{\tau}\right\}_{\tau=0}^{T-1}$ be given by $\bar{\gamma}_{0}=\frac{1}{\lambda b}$ and $\bar{\gamma}_{\tau+1}=\frac{\left(\bar{\gamma}_{\tau}+\widetilde{\pi}\right)}{1+\widetilde{\pi}}$ : given $\lambda b<1$, the sequence is monotonically decreasing and it defines a corresponding sequence of intervals $\left[\bar{\gamma}_{\tau+1}, \bar{\gamma}_{\tau}\right)$, for all $\tau$. By the productivity of $A$, the size of the intervals $\left[\bar{\gamma}_{\tau}, \bar{\gamma}_{\tau-1}\right)$ decreases with $\tau$ and tends to zero, with $\bar{\gamma}_{\tau} \rightarrow 1$ as $\tau \rightarrow \infty$. The sequence of intervals $\left[\bar{\gamma}_{\tau+1}, \bar{\gamma}_{\tau}\right)$ identifies a partition of the theoretically relevant set of aggregate productive endowments, given by the interval $\left(1, \frac{1}{\lambda b}\right]$.

Theorem 6 proves the existence of a SGP.

Theorem 6 Let $\beta \in\left(\frac{1}{\left(1+\pi^{\prime}\right)}, 1\right], \omega_{0}=\gamma_{0} N_{w} A(1-A)^{-1} b$, and $\gamma_{0}>1$. If $\gamma_{0} \in\left[\bar{\gamma}_{\tau+1}, \bar{\gamma}_{\tau}\right)$ and $\bar{\gamma}_{\tau}>\frac{\beta \tilde{\pi}}{\beta(1+\widetilde{\pi})-1}$, with $\tau \geqq 1$, then the vector $(p, \mathbf{1})$ with $\pi_{t}=\frac{\widetilde{\pi}\left(\gamma_{t}-1\right)}{\gamma_{t}}$, all $t, \tau \geqq t \geqq 0$, with $\gamma_{t+1}=\left(1+\pi_{t}\right) \gamma_{t}$, all $t \leqq \tau-1$, and $\pi_{t}=\pi_{\beta}$, all $t, T-1 \geqq t \geqq \tau+1$, is a SGP for $E_{0}$ with $\omega_{t+1}=\left(1+\pi_{t}\right) \omega_{t}$, all $t \leqq \tau-1, \omega_{\tau+1}=\left(1+g_{\tau}\right) \omega_{\tau}$, with $g_{\tau} \in\left(0, \pi_{\tau}\right]$, and $\omega_{t+1}=\omega_{t}$, all $t$, $T-1 \geqq t \geqq \tau+1$.

By Theorem 6, if initial aggregate endowments are greater than the minimum amount necessary to guarantee workers' subsistence $\left(\gamma_{0}>1\right)$, then the existence of a SGP can be proved for a range of values of the rate of time preference $\left(\beta \in\left(\frac{1}{\left(1+\pi^{\prime}\right)}, 1\right]\right)$. In equilibrium, there exists a period $\tau$ such that the economy accumulates and reaches the steady state in $\tau$ periods. The value of $\tau$ is determined by the initial amount of aggregate capital: if aggregate endowments are such that $\gamma_{0} \in$ $\left[\bar{\gamma}_{\tau+1}, \bar{\gamma}_{\tau}\right)$, then it takes $\tau$ periods for the economy to reach the steady state.

The dynamic path of the economy can thus be divided into two parts: in the first $\tau$ periods, capital grows at the maximum rate, which coincides with the profit rate $\left(\gamma_{t+1}=\left(1+\pi_{t}\right) \gamma_{t}\right.$, or, equivalently, $\left.\omega_{t+1}=\left(1+\pi_{t}\right) \omega_{t}\right)$. By construction, if $\gamma_{0} \in\left[\bar{\gamma}_{\tau+1}, \bar{\gamma}_{\tau}\right)$, then $\gamma_{1} \in\left[\bar{\gamma}_{\tau}, \bar{\gamma}_{\tau-1}\right)$, and so on. In turn, in each period $t$, the profit rate, and therefore production prices, are determined by aggregate endowments $\left(\pi_{t}=\frac{\widetilde{\pi}\left(\gamma_{t}-1\right)}{\gamma_{t}}\right)$. Thus, as aggregate capital grows, profits and labor expended increase over time. Yet, by construction $\gamma_{t} \lambda b<1$ for all periods $t \leqq \tau-1$, there is an excess supply of labor, and workers' consumption remains at the subsistence level. ${ }^{41}$

\footnotetext{
${ }^{40}$ Theorem 4 is reminiscent of the so-called Cambridge equation according to which $g_{t}=s \pi_{t}$ since, at least in some periods, the capitalists savings rate is equal to one and the growth rate is equal to the profit rate.

${ }^{41}$ Observe that in the first part of the SGP, the economy displays positive profits and accumulates at the maximal rate even if $\gamma_{0} \lambda b \leq 1$ and $\beta=1$. This is consistent with Theorem 3(iii), which rules out positive profits (and exploitation) at stationary equilibria if $\beta=1$.
} 
Once the steady state is reached, however, full employment prevails $\left(\gamma_{t} \lambda b=1\right)$, profits remain constant at the value that makes capitalists indifferent between accumulating or decumulating $\left(\pi_{t}=\pi_{\beta}\right)$, and workers' consumption exceeds subsistence. ${ }^{42}$ If $\beta<1$, UE exploitation is a persistent phenomenon; if $\beta=1$, it disappears. By construction, in every period (both when the economy accumulates, and at the steady state), all capitalists use up their entire wealth.

These results confirm the main conclusions of section 5 . Only if $\beta<1$ can overaccumulation - leading to the disappearance of UE exploitation - be ruled out in equilibrium (Proposition 2). ${ }^{43}$ Moreover, if $\beta=1$, inequalities, UE exploitation, and profits may well disappear after a finite number of periods, both at a SRS (Theorem 3) and at a SGP (Theorem 4), even if capital remains scarce in the sense of Definition 2. Instead, if agents discount the future, inequalities and UE exploitation can be persistent even in paths with capital accumulation (Theorem 4). The crucial role of time preference, as opposed, e.g., to capital scarcity, is further confirmed by the fact that if $\beta<1$, then the steady state value of the profit rate (and thus, by Theorem 1 , the rate of UE exploitation) is a positive function of $\beta$ (Theorem 4(ii)).

These results suggest that the role of asset inequalities (and competitive markets) in generating persistent inequalities and persistent UE exploitation in accumulating economies should be reconsidered. For asset inequalities are indeed necessary for the emergence of UE exploitation, but they are not sufficient for its persistence, which raises some doubts on the claim that exploitation can be reduced - either positively or normatively - to a purely distributive phenomenon (Roemer [54], p.2).

One may object that Theorems 3 and 4 actually prove that time discounting by capitalists is the missing ingredient necessary to guarantee the persistence of exploitation and inequalities, including in economies that accumulate and eventually reach a steady state. From this perspective, Theorem 3 and 4 can actually be seen as providing a dynamic generalization of Roemer's theory: provided agents discount the future, asset inequalities and competitive markets generate persistent UE exploitation.

Although this interpretation is certainly legitimate, we are not convinced that an explanation of persistent inequalities (in income and in the exchange of labor) that crucially relies on a strictly positive rate of time preference is the most promising or theoretically satisfactory. Furthermore, as argued in detail in Veneziani [62], if exploitation theory is understood as a distinctive approach in normative economics, then the significance of a purely subjective factor such as time preference is even less obvious both in general (see, for example, the classic analysis by Rawls [48]), and specifically in the context of exploitation theory. At the very least, we would argue, it is worth exploring alternative explanations, focusing on the structural features of capitalist economies.

In the rest of this section, we move a first step in this direction and consider the interaction between technical change and labor market conditions and institutions in shaping distributive outcomes and the equilibrium growth paths of capitalist economies.

To be specific, we consider the role of technical change and unemployment in creating the conditions for inequalities and exploitation to persist. As Dosi et al ([15], p.164) have forcefully noted, "Unemployment is a persistent and structural phenomenon of capitalist economies" of such relevance that "other possible stylized facts are in comparison second order ones". Theoretically, in Marx, unemployment is seen as a structural feature of capitalism, whose role is to discipline workers and to restrain wages from rising and, in turn, labor-saving technical change plays a key role in guaranteeing the persistence of a reserve army of the unemployed by increasing labor productivity.

In our model, the disappearance of exploitation derives from an initial excess supply of labor which is rapidly absorbed owing to accumulation. The introduction of labor saving technical progress should avoid this: by increasing labor productivity, technical progress may allow labor

\footnotetext{
${ }^{42}$ Given $\gamma_{0}>1$, this follows from the assumption that $\beta>\frac{1}{\left(1+\pi^{\prime}\right)}$, which implies that $p_{\beta} b<1$ and therefore workers can consume above subsistence.

${ }^{43}$ Thus, Devine and Dymsky's [14] result can only be an equilibrium if $\beta=1$.
} 
supply to be persistently higher than labor demand. ${ }^{44}$

To be specific, we take "the Schumpeterian view that the creation of new ideas largely occurs at an autonomous rate" (Shell [58], p.67) and assume that the amount of labor directly needed in production declines geometrically over time. ${ }^{45}$

Assumption 2 (A2): At all $t, L_{t+1}=\delta L_{t}, \delta \in(0,1)$, with $L_{0}>0$ given.

Under (A2), all the results in Section 4 hold, once $L_{t}$ is substituted for $L$. Then, Theorem 7 provides sufficient conditions for the existence of a RS with persistent exploitation.

Theorem 7 Assume (A2). Let $\omega_{0}=\gamma_{0} N_{w} A(1-A)^{-1} b, \gamma_{0}>1$, and $\gamma_{0} \lambda_{0} b \leqq 1$. Let $\delta(1+\tilde{\pi}) \leqq 1$ and $\beta\left[1+\frac{\widetilde{\pi}\left(\gamma_{0}-1\right)}{\gamma_{0}}\right] \geqq 1$. The price vector $(p, \mathbf{1})$ with $\pi_{0}=\frac{\widetilde{\pi}\left(\gamma_{0}-1\right)}{\gamma_{0}}$ and $\pi_{t+1}=\frac{\pi_{t}(1+\widetilde{\pi})}{\left(1+\pi_{t}\right)}$, all $t$, $T-2 \geqq t \geqq 0$, is a $R S$ for $E_{0}$ with $L_{t} y_{t}<N_{w}$, all $t>0$, and $\omega_{t+1}=\left(1+\pi_{t}\right) \omega_{t}$, all $t, T-2 \geqq t \geqq 0$.

Theorem 7 provides a complete characterization of dynamic general equilibrium paths with sustained growth and persistent - indeed, increasing - UE exploitation and inequality. Consider economies with aggregate initial assets above the minimum barely sufficient to guarantee workers' subsistence $\left(\gamma_{0}>1\right)$, but below the level that would make aggregate labor demand higher than aggregate labor supply $\left(\gamma_{0} \lambda_{0} b \leqq 1\right)$. If technical change is strong enough $(\delta(1+\widetilde{\pi}) \leqq 1)$ and agents are not too impatient $\left(\beta\left[1+\frac{\tilde{\pi}\left(\gamma_{0}-1\right)}{\gamma_{0}}\right] \geqq 1\right)$ then there exists an equilibrium with positive - indeed, increasing - profits, and by Theorem 1, UE exploitation in every period, as $\gamma_{0}>1$ implies $\pi_{0}>0$ and $\tilde{\pi}>\pi_{t}$, all $t$, implies $\pi_{t+1}>\pi_{t}$, all $t$. Along the equilibrium path, the economy grows at the maximal rate, $\pi_{t}$, but labor demand remains lower than labor supply $\left(L_{t} y_{t}<N_{w}\right)$.

Theorem 7 highlights an interesting mechanism that may contribute to the persistence of exploitation and inequalities in capitalist economies. For it shows that labor-saving technical progress allows the economy to settle on a "golden rule" growth path with persistent exploitation even if $\beta=1$. The increase in labor productivity - a long run historical tendency of capitalist economies (Acemoglu [1]) - ensures that labor remains in excess supply even along a growth path with maximal accumulation, thus countering all tendencies for profits and exploitation to disappear. Indeed, as the nominal wage rate is normalized to one and employment $L_{t} y_{t}$ either does not grow, or grows more slowly than national income $p_{t}(1-A) y_{t}$, Theorem 7 implies that the wage share in national income, $\frac{L_{t} y_{t}}{p_{t}(1-A) y_{t}}$, tends to fall steadily over time tending to zero in the long run as in Mookherjee and Ray [36].

\section{Conclusion}

In this paper, an intertemporal model with heterogeneous optimizing agents is set up to analyze the relation between inequalities - in income, wealth and labor exchanged, - and accumulation. We have argued that the concept of exploitation as the unequal exchange of labor is well defined, and it can be interpreted as capturing normatively relevant inequalities in well-being freedom. We have generalized the well-known correspondence between the existence of exploitation and positive profits to the dynamic economy. This provides a link between exploitation and the functional income distribution, but it also suggests that inequalities (both in wealth holdings and in the exchange of labor) are necessary for accumulation in capitalist economies.

\footnotetext{
${ }^{44}$ The relevance of exogenous growth in the labor force, heterogeneous preferences, and/or labor-saving technical progress in making exploitation persistent is stressed by Skillman [59]. Observe that, for the sake of simplicity, and without significant loss of generality we do not assume that technical change is capital using, in addition to being labor saving, and thus the capital coefficient $A$ remains constant.

${ }^{45}$ In other words, we are considering technology as "stemming from advances in science or from the behavior of entrepreneurs driven by a variety of nonprofit motives" (Acemoglu [2], p.11). This is just to focus on one key channel through which technical change affects distributive outcomes and growth, namely by preserving capital scarcity. We discuss more complex interactions between technical change and distributive conflict in the concluding section.
} 
We have also explored the mechanisms underlying the persistence of inequalities and exploitation in accumulating economies, and the relation between asset inequalities and exploitation. We have shown that asset inequalities are a fundamental feature of capitalist economies, and a key determinant of its long-run dynamics, but contrary to Roemer's $[52,54]$ seminal theory, it is unclear that exploitation can be reduced to wealth inequalities. For, differential ownership of productive assets is necessary for the emergence of exploitation but it is not sufficient for it to persist in equilibrium, even if capital does not become abundant.

Our analysis thus suggests a more complex role for asset inequalities in generating persistent UE exploitation and inequalities. The relevance of wealth inequalities, we believe, is best understood in conjunction with the asymmetric relations of power that characterize capitalist economies, the mechanisms that ensure the scarcity of capital, and the structural constraints that the differential ownership of productive assets imposes on aggregate investment, technical change, unemployment, and so on. From this perspective, Theorem 7 is the most promising result. For, the analysis of the economy with technical progress highlights a mechanism that may contribute to explain the persistence of UE exploitation and inequalities. In the long-run labor-saving technical progress tends to reduce the demand for labor, thus creating the conditions for an excess supply of labor, which restrains wages from rising, which in turn allows UE exploitation and inequalities to persist.

To be sure, Theorem 7 incorporates a rather simple mechanism through which exploitation and inequalities are reproduced over time, namely exogenous, labor-saving technical progress. One promising line for further research, from this perspective, would be the analysis of the endogenous competitive processes leading capitalists to introduce new cost-reducing techniques in general economies. As Bidard [12] has shown, in the multi-sectoral linear production model the adoption of cost-reducing technical change would involve a rather complex process under capitalist competition, which cannot be fully captured in the one-sector model in section 6. Further, Yoshihara and Veneziani [68] have recently proved that some types of cost-reducing technical change do not allow exploitation to persist. Another promising line for further research in this vein would be the introduction of endogenous R\&D investment, as in the literature on directed technical change (see Kennedy [28] and, more recently, Acemoglu [1,3]). One aspect of these strands of literature that is particularly congenial to our research agenda is the explicit relation between the functional income distribution and innovations, and the role of technical change in distributive conflicts. ${ }^{46}$

Another interesting extension of our analysis would be the construction of an index of the intensity of exploitation at the individual level, measuring the discrepancy between labor contributed and labor received by each agent, and then analyze the dynamics of the distribution of the exploitation intensity index over time, as the economy accumulates. Given that our concept of exploitation is based on empirically measurable magnitudes (labor expended, technology, income, and consumption), this approach might yield novel insights on the relation between inequality and growth. We leave these issues for further research.

\section{A Proofs of the Main Theorems}

\section{Proof of Theorem 1:}

Proof. Part (i). Consider any $t$. By Definition 1(ii) and (v), at a RS $L y_{t}=z_{t}=p_{t} c_{t}^{w}$. Then, noting that $c_{t}^{w}>\mathbf{0}$, by Lemma $2, L y_{t}>\lambda c_{t}^{w}$ if and only if $\pi_{t}>0$.

Part (ii). The result follows from part (i), since $L y_{t}-\lambda c_{t}^{w} \geqq 0$, all $t$.

Part (iii). Let $(\mathbf{p}, 1)$ be a RS for $E_{0}$ with $\pi_{t}>0$. For this $\pi_{t}>0$, the equilibrium price vector $p_{t}>\mathbf{0}$ is uniquely determined by $p_{t} \equiv L\left(I-\left(1+\pi_{t}\right) A\right)^{-1}$. Correspondingly, the aggregate demand vector $c_{t}^{w}>\mathbf{0}$ is also uniquely determined. As argued in part (i), we observe that $L y_{t}=p_{t} c_{t}^{w}$ at the RS. Moreover, $L y_{t}=L A^{-1} \omega_{t}$ by Lemma 1 . Given $\omega_{t}>\mathbf{0}$, let $\left(p_{t}^{\prime}, 1\right)$ be an equilibrium

\footnotetext{
${ }^{46}$ We are grateful to an anonymous referee for this suggestion.
} 
price associated with $\pi_{t}^{\prime}>0$, such that the associated $y_{t}, c_{t}^{c \prime}, c_{t}^{w \prime}$, and $s_{t}^{\prime}$ satisfy the conditions of Definition 1. Then, again $L A^{-1} \omega_{t}=p_{t}^{\prime} c_{t}^{w \prime}$ holds. Therefore, by Definition 3, the corresponding exploitation rate is uniquely determined by $e_{t}^{\prime}=\frac{p_{t}^{\prime} c_{t}^{w \prime}}{\lambda c_{t}^{w \prime}}-1$.

To show the monotone increasing relationship of $\pi\left(e_{t}\right)=\pi_{t}$ at period $t$, we need to verify that $\pi_{t} \gtreqless \pi_{t}^{\prime}$ if and only if $e_{t} \gtreqless e_{t}^{\prime}$. Let $\pi_{t}^{\prime}>\pi_{t}$. Then, it implies $p_{t}^{\prime} \equiv L\left(I-\left(1+\pi_{t}^{\prime}\right) A\right)^{-1}>p_{t}$. Then, given that $p_{t} c_{t}^{w}=L A^{-1} \omega_{t}=p_{t}^{\prime} c_{t}^{w \prime}, p_{t}^{\prime}>p_{t}$ implies $c_{t}^{w \prime} \leq c_{t}^{w}$ by (A1). Therefore, $\lambda c_{t}^{w \prime}<\lambda c_{t}^{w}$ holds, which implies that $e_{t}^{\prime}>e_{t}$. Conversely, to show that $e_{t}^{\prime}>e_{t}$ implies $\pi_{t}^{\prime}>\pi_{t}$, assume $\pi_{t}^{\prime} \leqq \pi_{t}$. If $\pi_{t}^{\prime}<\pi_{t}$, then $e_{t}<e_{t}^{\prime}$ holds, as argued for the case of $\pi_{t}^{\prime}>\pi_{t}$. Therefore, let $\pi_{t}^{\prime}=\pi_{t}$. Then, it implies that $p_{t}^{\prime}=L\left(I-\left(1+\pi_{t}\right) A\right)^{-1}=p_{t}$, and so $c_{t}^{w \prime}=c_{t}^{w}$. Thus, $e_{t}=e_{t}^{\prime}$ holds, as $\lambda c_{t}^{w \prime}=\lambda c_{t}^{w}$ holds. In summary, we can find a monotone increasing function $\pi\left(e_{t}\right)=\pi_{t}$.

\section{Proof of Theorem 2:}

Proof. Part (i). Write $M P^{\nu}$ using dynamic recursive optimization theory. Let $\mathcal{W} \subseteq \mathbb{R}_{+}^{n}$ be the state space with generic element $\omega$. For any $(\mathbf{p}, 1)$, let $\Psi: \mathcal{W} \rightarrow \mathcal{W}$ be the feasibility correspondence: $\Psi\left(\omega_{t}^{\nu}\right)=\left\{\omega_{t+1}^{\nu} \in \mathcal{W}: p_{t} \omega_{t+1}^{\nu} \leqq\left(1+\pi_{t}\right) p_{t} \omega_{t}^{\nu}\right\}$. Let

$$
\Pi\left(\omega_{0}^{\nu}\right)=\left\{\omega^{\nu}: \omega_{t+1}^{\nu} \in \Psi\left(\omega_{t}^{\nu}\right), \text { all } t, \omega_{T}^{\nu} \geqq \omega_{0}^{\nu}, \text { and } \omega_{0}^{\nu} \text { given }\right\} .
$$

Let $\Phi=\left\{\left(\omega_{t}^{\nu}, \omega_{t+1}^{\nu}\right) \in \mathcal{W} \times \mathcal{W}: \omega_{t+1}^{\nu} \in \Psi\left(\omega_{t}^{\nu}\right)\right\}$ be the graph of $\Psi$. By the homogeneity of $\phi$, if $\pi_{t}=\pi_{\beta}$, all $t$, then the one-period return function $F: \Phi \rightarrow \mathbb{R}_{+}$at $t$ is $F\left(\omega_{t}^{\nu}, \omega_{t+1}^{\nu}\right)=$ $\frac{\phi\left(c_{\beta}\right)\left[\left(1+\pi_{\beta}\right) p_{\beta} \omega_{t}^{\nu}-p_{\beta} \omega_{t+1}^{\nu}\right]}{p_{\beta} c_{\beta}}$. Then, $M P^{\nu}$ can be written as

$$
C\left(\omega_{0}^{\nu}\right)=\max _{\omega^{\nu} \in \Pi\left(\omega_{0}^{\nu}\right)} \sum_{t=0}^{T-1} \beta^{t} \frac{\phi\left(c_{\beta}\right)\left[\left(1+\pi_{\beta}\right) p_{\beta} \omega_{t}^{\nu}-p_{\beta} \omega_{t+1}^{\nu}\right]}{p_{\beta} c_{\beta}} .
$$

Since $\Psi\left(\omega_{t}^{\nu}\right) \neq \varnothing$, all $\omega_{t}^{\nu} \in \mathcal{W}$, and $F$ is continuous, concave, and bounded below by $0, M P^{\nu}$ is well defined.

2. By construction, $\left(1+\pi_{\beta}\right) \beta=1$ and $M P^{\nu}$ reduces to

$$
C\left(\omega_{0}^{\nu}\right)=\max _{\omega^{\nu} \in \Pi\left(\omega_{0}^{\nu}\right)} \phi\left(c_{\beta}\right)\left[\frac{\left(1+\pi_{\beta}\right) p_{\beta} \omega_{0}^{\nu}}{p_{\beta} c_{\beta}}-\beta^{T-1} \frac{p_{\beta} \omega_{T}^{\nu}}{p_{\beta} c_{\beta}}\right] .
$$

Therefore, any $\omega^{\nu} \in \Pi\left(\omega_{0}^{\nu}\right)$ such that $\omega_{T}^{\nu}=\omega_{0}^{\nu}$ is optimal and $C\left(\omega_{0}^{\nu}\right)$ follows by noting that $\beta<1$.

Part (ii). The result follows from $M P^{\nu}$, given that $\omega_{T}^{\nu} \geqq \omega_{0}^{\nu}$.

\section{Proof of Theorem 3:}

Proof. Part (i). 1. (Optimal $\xi^{\nu}$.) By the Perron-Frobenius theorem $\pi^{\prime}$ exists and $\pi^{\prime} \in(0, \tilde{\pi})$. (The possibility that $\pi^{\prime}=0$ is ruled out by the condition $\gamma_{0} \lambda b=L\left[I-\left(1+\pi^{\prime}\right) A\right]^{-1} b$ given $\gamma_{0}>1$.) If $\pi^{\prime}=\pi_{\beta}, c_{\beta}=k b$, some $k>0$, and $\pi_{t}=\pi^{\prime}$, all $t$, by Theorem 2 , any $\xi^{\nu}$ such that $s_{t}^{\nu}=\mathbf{0}$, $p_{\beta} A y_{t}^{\nu}=p_{\beta} \omega_{0}^{\nu}$, and $c_{t}^{\nu}=h_{t}^{\nu} b$ with $h_{t}^{\nu}=\frac{\pi^{\prime} p_{\beta} \omega_{0}^{\nu}}{p_{\beta} b}$, all $t$, solves $M P^{\nu}$, for all $\nu \in \mathcal{N}_{c}$.

2. (Capital market.) Hence, it is possible to choose $\left(y^{\nu}\right)_{\nu \in \mathcal{N}_{c}}$ such that at all $t, p_{\beta} A y_{t}^{\nu}=p_{\beta} \omega_{0}^{\nu}$, all $\nu$, and $y_{t}=A^{-1} \omega_{0}$.

3. (Labor market and optimal $\xi^{\eta}$.) Since $L y_{t}=\gamma_{0} \lambda b N_{w}<N_{w}$, all $t$, for all $\eta \in \mathcal{N}_{w}$ assign actions $z_{t}^{\eta}=\widehat{z}_{t}^{\eta}=\gamma_{0} \lambda b$, all $t$; then by construction $\gamma_{0} \lambda b=p_{\beta} b$, and thus $c_{t}^{\eta}=b$, all $t$. Hence, these actions solve $M P^{\eta}$ for all $\eta$, with $L y_{t}=z_{t}$, all $t$.

4. (Final goods market.) Definition 1(iii) is satisfied because, at all $t:(I-A) y_{t}=\gamma_{0} N_{w} b$, $c_{t}^{w}=N_{w} b$, and $c_{t}^{c}=h_{t}^{c} b$, where $h_{t}^{c}=\sum_{\nu \in \mathcal{N}_{c}} h_{t}^{\nu}$, and so $h_{t}^{c} p_{\beta} b=\gamma_{0} N_{w}\left[p_{\beta}-\lambda\right] b$, or $h_{t}^{c}=N_{w}\left(\gamma_{0}-1\right)$.

Part (ii). 1. (Optimal $\xi^{\nu}$.) By the Perron-Frobenius theorem $\pi^{\prime}$ exists and $\pi^{\prime} \in(0, \tilde{\pi})$. Thus $\pi_{\beta} \in(0, \tilde{\pi})$. If $\pi_{t}=\pi_{\beta}$, all $t$, by Theorem 2 , any $\xi^{\nu}$ such that $s_{t}^{\nu}=\mathbf{0}, p_{\beta} A y_{t}^{\nu}=p_{\beta} \omega_{0}^{\nu}$, and $c_{t}^{\nu}=h_{t}^{\nu} b$ with $h_{t}^{\nu} p_{\beta} b=\pi_{\beta} p_{\beta} \omega_{0}^{\nu}$, all $t$, solves $M P^{\nu}$, for all $\nu \in \mathcal{N}_{c}$. 
2. (Capital market.) Hence, it is possible to choose $\left(y^{\nu}\right)_{\nu \in \mathcal{N}_{c}}$ such that at all $t, p_{\beta} A y_{t}^{\nu}=p_{\beta} \omega_{0}^{\nu}$, all $\nu$, and $y_{t}=A^{-1} \omega_{0}$.

3. (Labor market; optimal $\xi^{\eta}$.) Since $L y_{t}=N_{w}$, all $t$, assign actions $z_{t}^{\eta}=\widehat{z}_{t}^{\eta}=1$ and $c_{t}^{\eta}=h_{t}^{\eta} b$ with $h_{t}^{\eta}=1 / p_{\beta} b$, all $t$, to all $\eta \in \mathcal{N}_{w}$. Since $\pi_{\beta} \in\left(0, \pi^{\prime}\right]$ then $1 / \lambda b>h_{t}^{\eta} \geqq 1$, all $t, \eta$. Hence, these actions solve $M P^{\eta}$ for all $\eta$, with $L y_{t}=z_{t}$, all $t$.

4. (Final goods market.) Definition 1(ii) is met because, at all $t,(I-A) y_{t}=\gamma_{0} N_{w} b$ while $c_{t}^{w}=$ $N_{w} b / p_{\beta} b$ and $c_{t}^{c}=\sum_{\nu \in \mathcal{N}_{c}} h_{t}^{\nu} b$, where $\sum_{\nu \in \mathcal{N}_{c}} h_{t}^{\nu} p_{\beta} b=\pi_{\beta} p_{\beta} \omega_{0}$, or $\sum_{\nu \in \mathcal{N}_{c}} h_{t}^{\nu} p_{\beta} b=\gamma_{0} N_{w}\left[p_{\beta}-\lambda\right] b$.

Part (iii). 1. If $\gamma_{0} \lambda b=1$, existence is proved as in part (ii) with $z_{t}^{\eta}=\widehat{z}_{t}^{\eta}=1$ and $h_{t}^{\eta}=1 / \lambda b$, all $\eta \in \mathcal{N}_{w}$, and all $t$. If $\gamma_{0} \lambda b<1$, existence is proved as in part (i) with $y_{t}=\left(1 / \gamma_{0}\right) A^{-1} \omega_{0}$ and $L y_{t}=\lambda b N_{w}$, all $t, z_{t}^{\eta}=\widehat{z}_{t}^{\eta}=\lambda b$ and $c_{t}^{\eta}=b$, all $\eta \in \mathcal{N}_{w}$, and all $t$. In both cases, as shown in parts (i) and (ii), $p_{t} A y_{t}^{\nu}=p_{t} \omega_{t}^{\nu}$, at all $t$ and for all $\nu \in \mathcal{N}_{c}$.

2. Suppose, by contradiction, that there is a SRS with $\pi_{t}>0$, some $t$. Then, using the same perturbational argument as in the proof of Lemma 3, it is immediate to prove that it must be $\pi_{t-1}=\pi_{t+1}=0$ (observe that since $\beta=1, \pi_{t+1}>0$ implies $\beta\left(1+\pi_{t+1}\right)>1$ ). Therefore noting that $p_{t}=\left(1+\pi_{t}\right) p_{t} A+w_{t} L$, and $w_{t}=1$ all $t$, it follows that $p_{t-1}=p_{t+1}=\lambda>\mathbf{0}$, for any $0 \leqq t-1<t+1 \leqq T-1$. Then, for all $\nu \in \mathcal{N}_{c}$, there is no optimal $\xi^{\nu}$ such that $s_{t}^{\nu}=\mathbf{0}$ and $c_{t-1}^{\nu}=c_{t+1}^{\nu}=c_{t}^{\nu}$, a contradiction.

\section{Proof of Theorem 4:}

Proof. Part (ii). 1. Consider capitalist $\nu$ 's program $M P^{\nu}$ recursively: at all $t$, the functional equation is $C_{t}\left(\omega_{t}^{\nu}\right)=\max _{\omega_{t+1}^{\nu} \in \Psi\left(\omega_{t}^{\nu}\right)}\left[\left(1+\pi_{t}\right) \omega_{t}^{\nu}-\omega_{t+1}^{\nu}\right]+\beta C_{t+1}\left(\omega_{t+1}^{\nu}\right)$. At $T-1$, since $C_{T}\left(\omega_{T}^{\nu}\right)=0$ for all $\omega_{T}^{\nu}$, optimality requires $\omega_{T}^{\nu}=\omega_{0}^{\nu}$ and $C_{T-1}\left(\omega_{T-1}^{\nu}\right)=\left[\left(1+\pi_{T-1}\right) \omega_{T-1}^{\nu}-\omega_{0}^{\nu}\right]$. Therefore at $T-2$, $C_{T-2}\left(\omega_{T-2}^{\nu}\right)=\max _{\omega_{T-1}^{\nu} \in \Psi\left(\omega_{T-2}^{\nu}\right)}\left[\left(1+\pi_{T-2}\right) \omega_{T-2}^{\nu}-\omega_{T-1}^{\nu}\right]+\beta C_{T-1}\left(\omega_{T-1}^{\nu}\right)$.

2. Suppose $\beta<1$ and $\pi_{t}>0$, all $t, T-2 \geqq t \geqq t^{\prime}$. Because $\pi_{T-2}>0$, if $\beta\left(1+\pi_{T-1}\right) \neq 1$ then $\omega_{T-1}^{\nu} \neq \omega_{T-2}^{\nu}$, all $\nu \in \mathcal{N}_{c}$, and $\omega_{T-1} \neq \omega_{T-2}$. Hence, $\beta\left(1+\pi_{T-1}\right)=1$ and $C_{T-2}\left(\omega_{T-2}^{\nu}\right)=$ $\left[\left(1+\pi_{T-2}\right) \omega_{T-2}^{\nu}-\beta \omega_{0}^{\nu}\right]$. Iterating backwards, if $\omega_{t+1}=\omega_{t}$, all $t, T-2 \geqq t \geqq t^{\prime}$, then $\beta\left(1+\pi_{t+1}\right)=$ 1 , all $t, T-2 \geqq t \geqq t^{\prime}$, which implies $C_{t^{\prime}}\left(\omega_{t^{\prime}}^{\nu}\right)=\left[\left(1+\pi_{t^{\prime}}\right) \omega_{t^{\prime}}^{\nu}-\beta^{T-1-t^{\prime}} \omega_{0}^{\nu}\right]$.

3. Suppose $\beta=1$. Suppose, contrary to the statement, that $\pi_{t}>0$ and $\pi_{t+j}>0$, some $t$, $T-2 \geqq t \geqq t^{\prime}$, and $j>0$. Since $\pi_{t}>0$, then $c_{t}^{\nu}=0$, all $\nu \in \mathcal{N}_{c}$, is not possible, or else $\omega_{t+1} \neq \omega_{t}$, and since $\pi_{t+j}>0$ then $\left(1+\pi_{t+j}\right)>1$, and there is a feasible perturbation $\mathrm{d} s_{t}^{\nu}=-\mathrm{d} s_{t+j}^{\nu}>0$, with $\mathrm{d} s_{l}^{\nu}=0$ all $l \neq t, t+j$, that increases $\nu$ 's welfare, contradicting optimality.

Part (i). 1. Suppose that $(\mathbf{p}, 1)$ is a SGP for $E_{0}$. Then by definition there is a $t^{\prime}>0$ and a sequence $\left\{g_{t}\right\}_{t=0}^{t^{\prime}-2}$ such that $\omega_{t+1}=\left(1+g_{t}\right) \omega_{t}, g_{t}>0$, all $t, 0 \leqq t<t^{\prime}-1$. For all $\nu \in \mathcal{N}_{c}$, $c_{t}^{\nu}=\pi_{t} \omega_{t}^{\nu}-s_{t}^{\nu}$, all $t$. Therefore, summing over $\nu$ and noting that by definition $s_{t}=g_{t} \omega_{t}$, all $t$, it follows that $c_{t}^{c}=\left(\pi_{t}-g_{t}\right) \omega_{t}$, all $t$. Since $\omega_{t}=\gamma_{t} N_{w} A(1-A)^{-1} b$, all $t$, and noting that in the one good case $\widetilde{\pi}=\frac{1-A}{A}$, then $c_{t}^{c}=\left(\pi_{t}-g_{t}\right) \frac{\gamma_{t} N_{w} b}{\widetilde{\pi}}$, all $t$, or $g_{t}=\left[\pi_{t}-\left(\frac{c_{t}^{c} \widetilde{\pi}}{\gamma_{t} N_{w} b}\right)\right]$, all $t$.

2. By definition, $\left(p_{t}-\lambda\right)=\pi_{t} p_{t} A(1-A)^{-1}$, all $t$, or equivalently $\pi_{t}=\widetilde{\pi}\left(p_{t}-\lambda\right) / p_{t}$, all $t$. Hence, $g_{t}=\left[\frac{\left(p_{t}-\lambda\right)}{p_{t}}-\left(\frac{c_{t}^{c}}{\gamma_{t} N_{w} b}\right)\right] \tilde{\pi}$, all $t$. Moreover, observe that at a SGP with $L A^{-1} \omega_{t}=\gamma_{t} N_{w} \lambda b \leqq N_{w}$, all $t$, it must be $\gamma_{t} \lambda b<1$, all $t \leqq t^{\prime}-1$. By construction, this implies that at all $t \leqq t^{\prime}-1$, $z_{t}^{\eta}=\gamma_{t} \lambda b=p_{t} b$, for all $\eta \in \mathcal{N}_{w}$. Therefore $p_{t}=\gamma_{t} \lambda$, all $t \leqq t^{\prime}-1$, and the first part of the statement follows substituting the latter expression into the equation for $g_{t}$, and noting that $\frac{p_{t+1}}{p_{t}}=\frac{\gamma_{t+1}}{\gamma_{t}}=\frac{\omega_{t+1}}{\omega_{t}}$, for all $t<t^{\prime}-1$.

3. Suppose $\beta<1$. If $t^{\prime} \leqq 2$, then the statement holds vacuously. Hence, assume $t^{\prime}>2$. At $t=t^{\prime}-1, C_{t^{\prime}-1}\left(\omega_{t^{\prime}-1}^{\nu}\right)=\max _{\omega_{t^{\prime}}^{\nu} \in \Psi\left(\omega_{t^{\prime}-1}^{\nu}\right)}\left[\left(1+\pi_{t^{\prime}-1}\right) \omega_{t^{\prime}-1}^{\nu}-\omega_{t^{\prime}}^{\nu}\right]+\beta C_{t^{\prime}}\left(\omega_{t^{\prime}}^{\nu}\right)$, where $C_{t^{\prime}}\left(\omega_{t^{\prime}}^{\nu}\right)$ is as in step 2 of the proof of part (ii) for all $\nu \in \mathcal{N}_{c}$. Hence, at a SGP $\beta\left(1+\pi_{t^{\prime}}\right) \geqq 1$, or else $\omega_{t^{\prime}}^{\nu}=0$, all $\nu \in \mathcal{N}_{c}$. If $\beta\left(1+\pi_{t^{\prime}}\right)>1$, then $\omega_{t^{\prime}}^{\nu}=\left(1+\pi_{t^{\prime}-1}\right) \omega_{t^{\prime}-1}^{\nu}$, all $\nu$, and $g_{t^{\prime}-1}=\pi_{t^{\prime}-1}$. If $\beta\left(1+\pi_{t^{\prime}}\right)=1$, then $g_{t^{\prime}-1}$ is undetermined. In either case, $C_{t^{\prime}-1}\left(\omega_{t^{\prime}-1}^{\nu}\right)=\left[\beta\left(1+\pi_{t^{\prime}}\right)\left(1+\pi_{t^{\prime}-1}\right) \omega_{t^{\prime}-1}^{\nu}-\beta^{T-t^{\prime}} \omega_{0}^{\nu}\right]$, all $\nu \in \mathcal{N}_{c}$. 
4. Consider $t=t^{\prime}-2$. Again, at a SGP, it must be $\beta^{2}\left(1+\pi_{t^{\prime}}\right)\left(1+\pi_{t^{\prime}-1}\right) \geqq 1$, and $C_{t^{\prime}-2}\left(\omega_{t^{\prime}-2}^{\nu}\right)$ $=\left[\beta^{2}\left(1+\pi_{t^{\prime}}\right)\left(1+\pi_{t^{\prime}-1}\right)\left(1+\pi_{t^{\prime}-2}\right) \omega_{t^{\prime}-2}^{\nu}-\beta^{T-t^{\prime}+1} \omega_{0}^{\nu}\right]$, all $\nu \in \mathcal{N}_{c}$. If $\beta^{2}\left(1+\pi_{t^{\prime}}\right)\left(1+\pi_{t^{\prime}-1}\right)=1$, then by the previous step $\beta\left(1+\pi_{t^{\prime}-1}\right) \leqq 1$ : but then since by step 2 at a SGP $p_{t+1}>p_{t}$, all $t<t^{\prime}-1$, by definition it follows that $\beta\left(1+\pi_{t^{\prime}-2}\right)<1$. However, because $t^{\prime}>2$, by considering $C_{t^{\prime}-3}\left(\omega_{t^{\prime}-3}^{\nu}\right)$, it immediately follows that $\omega_{t^{\prime}-2}^{\nu}=0$, all $\nu \in \mathcal{N}_{c}$, violating the definition of SGP. Therefore, it must be $\beta^{2}\left(1+\pi_{t^{\prime}}\right)\left(1+\pi_{t^{\prime}-1}\right)>1, \omega_{t^{\prime}-1}^{\nu}=\left(1+\pi_{t^{\prime}-2}\right) \omega_{t^{\prime}-2}^{\nu}$, all $\nu$, and $g_{t^{\prime}-2}=\pi_{t^{\prime}-2}$. This argument can be iterated backwards for all $t, 0<t<t^{\prime}-1$, showing that $\omega_{t+1}^{\nu}=\left(1+\pi_{t}\right) \omega_{t}^{\nu}$, all $\nu$, and all $t, 0<t<t^{\prime}-1$, and thus $g_{t}=\pi_{t}$, all $t, 0<t<t^{\prime}-1$.

5. Suppose $\beta=1$. A similar argument as in steps 3 and 4 applies noting that at all $t \leqq t^{\prime}-1$, $\pi_{t}>0$ implies $\beta\left(1+\pi_{t}\right)>1$, given part (ii).

\section{Proof of Theorem 5:}

Proof. 1. Take any $\nu \in \mathcal{N}_{c}$. Consider $M P^{\nu}$ recursively. At $T-1$, since $C_{T}\left(\omega_{T}^{\nu}\right)=0$, then $\omega_{T}^{\nu}=\omega_{0}^{\nu}$ is optimal and $C_{T-1}\left(\omega_{T-1}^{\nu}\right)=\left[\left(1+\pi_{T-1}\right) \omega_{T-1}^{\nu}-\omega_{0}^{\nu}\right]$. At $T-2, C_{T-2}\left(\omega_{T-2}^{\nu}\right)=\max _{\omega_{T-1}^{\nu} \in \Psi\left(\omega_{T-2}^{\nu}\right)}[(1+$ $\left.\left.\pi_{T-2}\right) \omega_{T-2}^{\nu}-\omega_{T-1}^{\nu}+\beta C_{T-1}\left(\omega_{T-1}^{\nu}\right)\right]$. Hence, if $\pi_{T-1}=\pi_{\beta}$ then any $\omega_{T-1}^{\nu} \geqq \omega_{T-2}^{\nu}$ is optimal and $C_{T-2}\left(\omega_{T-2}^{\nu}\right)=\left[\left(1+\pi_{T-2}\right) \omega_{T-2}^{\nu}-\beta \omega_{0}^{\nu}\right]$. Iterating backwards, if $\pi_{t}=\pi_{\beta}$, all $t, T-1 \geqq t \geqq \tau+1$, then at all $t, T-2 \geqq t \geqq \tau$, any $\omega_{t+1}^{\nu} \geqq \omega_{t}^{\nu}$ is optimal and $C_{\tau}\left(\omega_{\tau}^{\nu}\right)=\left[\left(1+\pi_{\tau}\right) \omega_{\tau}^{\nu}-\beta^{T-\tau-1} \omega_{0}^{\nu}\right]$. If $\tau=0$, the result is proved, noting that $C\left(\omega_{0}^{\nu}\right)=C_{0}\left(\omega_{0}^{\nu}\right)$.

2. If $\tau>0$, consider $\tau-1$. Since $C_{\tau-1}\left(\omega_{\tau-1}^{\nu}\right)=\max _{\omega_{\tau}^{\nu} \in \Psi\left(\omega_{\tau-1}^{\nu}\right)}\left[\left(1+\pi_{\tau-1}\right) \omega_{\tau-1}^{\nu}-\omega_{\tau}^{\nu}+\beta C_{\tau}\left(\omega_{\tau}^{\nu}\right)\right]$ and $\pi_{t}>\pi_{\beta}$, at the solution to $M P^{\nu}, \omega_{\tau}^{\nu}=\left(1+\pi_{\tau-1}\right) \omega_{\tau-1}^{\nu}$ and $C_{\tau-1}\left(\omega_{\tau-1}^{\nu}\right)=\left[\beta\left(1+\pi_{\tau}\right)\left(1+\pi_{\tau-1}\right) \omega_{\tau-1}^{\nu}-\right.$ $\left.\beta^{T-\tau} \omega_{0}^{\nu}\right]$. Iterating backwards, if $\pi_{t}>\pi_{\beta}$, all $t \leqq \tau$, at the solution to $M P^{\nu}, \omega_{t+1}^{\nu}=\left(1+\pi_{t}\right) \omega_{t}^{\nu}$, all $t \leqq \tau-1$, and the expression for $C\left(\omega_{0}^{\nu}\right)=C_{0}\left(\omega_{0}^{\nu}\right)$ follows.

\section{Proof of Theorem 6:}

Proof. 1. We begin by establishing three properties of the sequence $\left\{\gamma_{t}\right\}_{t=0}^{T-1}$.

1.1. At all $t \leqq \tau$, if $\gamma_{t} \in\left[\bar{\gamma}_{\tau+1-t}, \bar{\gamma}_{\tau-t}\right)$ and $\pi_{t}=\tilde{\pi} \frac{\left(\gamma_{t}-1\right)}{\gamma_{t}}$, then $\gamma_{t+1}=\left(1+\pi_{t}\right) \gamma_{t}$ implies $\gamma_{t+1} \in\left[\bar{\gamma}_{\tau-t}, \bar{\gamma}_{\tau-t-1}\right)$. To see this, note that at all $\tau, \bar{\gamma}_{\tau}=(1+\widetilde{\pi}) \bar{\gamma}_{\tau+1}-\tilde{\pi}$, while $\gamma_{t+1}=\left(1+\pi_{t}\right) \gamma_{t}$ and $\pi_{t}=\tilde{\pi} \frac{\left(\gamma_{t}-1\right)}{\gamma_{t}}$ implies $\gamma_{t+1}=\left(1+\tilde{\pi} \frac{\left(\gamma_{t}-1\right)}{\gamma_{t}}\right) \gamma_{t}=(1+\tilde{\pi}) \gamma_{t}-\tilde{\pi}$.

1.2. If $\gamma_{t} \in\left[\bar{\gamma}_{1}, \bar{\gamma}_{0}\right)=\left[\bar{\gamma}_{1}, \frac{1}{\lambda b}\right)$ and $\pi_{t}=\widetilde{\pi} \frac{\left(\gamma_{t}-1\right)}{\gamma_{t}}$, then there is a $g_{t} \in\left(0, \pi_{t}\right]$ such that $\gamma_{t+1}=$ $\left(1+g_{t}\right) \gamma_{t}$ implies $\gamma_{t+1}=1 / \lambda b$. To see this, note that, as in step $1.1, \bar{\gamma}_{0}=(1+\tilde{\pi}) \bar{\gamma}_{1}-\tilde{\pi}$. Therefore if $\gamma_{t}=\bar{\gamma}_{1}$ and $\pi_{t}=\widetilde{\pi} \frac{\left(\gamma_{t}-1\right)}{\gamma_{t}}$, then $g_{t}=\pi_{t}$ implies $\gamma_{t+1}=\bar{\gamma}_{0}$, and for all $\gamma_{t} \in\left(\bar{\gamma}_{1}, \bar{\gamma}_{0}\right), g_{t}=\pi_{t}$ implies $\gamma_{t+1}>\bar{\gamma}_{0}$, while $g_{t}=0$ implies $\gamma_{t+1}<\bar{\gamma}_{0}$.

1.3. If $\bar{\gamma}_{\tau}>\frac{\beta \tilde{\pi}}{\beta(1+\widetilde{\pi})-1}$, all $\tau \geqq 1$, then $\pi_{1}=\widetilde{\pi} \frac{\left(\gamma_{1}-1\right)}{\gamma_{1}}>\pi_{\beta}$, for all $\gamma_{1} \in\left[\bar{\gamma}_{\tau}, \bar{\gamma}_{\tau-1}\right)$. To see this, note that if $\gamma_{1}=\bar{\gamma}_{\tau}$ then $\pi_{1}=\tilde{\pi}\left(1-\frac{1}{\bar{\gamma}_{\tau}}\right)>\widetilde{\pi}\left(1-\frac{\beta(1+\widetilde{\pi})-1}{\beta \tilde{\pi}}\right)=\pi_{\beta}$, and $\pi_{1}$ is strictly increasing in $\gamma_{1}$.

2. Consider $(\mathbf{p}, 1)$ with $\pi_{t}=\widetilde{\pi} \frac{\left(\gamma_{t}-1\right)}{\gamma_{t}}$, all $t \leqq \tau$, where $\gamma_{t+1}=\left(1+\pi_{t}\right) \gamma_{t}$, all $t \leqq \tau-1$. Then $\pi_{0}=\widetilde{\pi} \frac{\left(\gamma_{0}-1\right)}{\gamma_{0}}$ and $\pi_{t+1}=\widetilde{\pi}\left(1-\frac{1}{\left(1+\pi_{t}\right) \gamma_{t}}\right)$, and $(\mathbf{p}, 1)$ is well defined.

3. (Optimal $\xi^{\nu}$; reproducibility.) By step 1.3, and noting that $\gamma_{0}>1$, under the assumptions of the Theorem, we have $\pi_{t}>\pi_{\beta}$, all $t \leqq \tau$. Hence, by Theorem $5, \omega_{t+1}^{\nu}=\left(1+\pi_{t}\right) \omega_{t}^{\nu}$, all $t \leqq \tau-1, \omega_{t+1}^{\nu}=\left(1+g_{t}\right) \omega_{t}^{\nu}$, with $g_{t} \in\left[0, \pi_{t}\right]$, all $t, T-1 \geqq t \geqq \tau$, and $\omega_{T}^{\nu}=\omega_{0}^{\nu}$ is optimal for all $\nu \in \mathcal{N}_{c}$. Therefore, for all $\nu \in \mathcal{N}_{c}$, we can choose an optimal $\xi^{\nu}$ such that $\omega_{t+1}^{\nu}=\left(1+\pi_{t}\right) \omega_{t}^{\nu}$, all $t \leqq \tau-1, \omega_{\tau+1}^{\nu}=\left(1+g_{\tau}\right) \omega_{\tau}^{\nu}$, with $g_{\tau}=\left(\frac{1}{\gamma_{\tau} \lambda b}-1\right) \in\left(0, \pi_{\tau}\right], \omega_{t}^{\nu}=\omega_{\tau+1}^{\nu}$, all $t, T-1 \geqq t \geqq \tau+1$, $\omega_{T}^{\nu}=\omega_{0}^{\nu} ; y_{t}^{\nu}=A^{-1} \omega_{t}^{\nu}$, all $t$; and $c_{t}^{\nu}=\left(1+\pi_{t}\right) \omega_{t}^{\nu}-\omega_{t+1}^{\nu}$, all $t$. (Observe that by steps 1.1 and 1.2 , $g_{\tau}=\left(\frac{1}{\gamma_{\tau} \lambda b}-1\right) \in\left(0, \pi_{\tau}\right]$ exists and $\gamma_{\tau+1}=\bar{\gamma}_{0}$.) Hence, parts (i) and (vi) of Definition 1 are met. 
4. (Capital market.) Because $y_{t}^{\nu}=A^{-1} \omega_{t}^{\nu}$, all $t$ and all $\nu \in \mathcal{N}_{c}$, then $y_{t}=A^{-1} \omega_{t}$, all $t$, and Definition 1(iv) is satisfied.

5. (Labor market; optimal $\xi^{\eta}$.) By construction, $\gamma_{0}<\bar{\gamma}_{1}<\bar{\gamma}_{0}=\frac{1}{\lambda b}$ and therefore $L y_{0}=$ $L A^{-1} \omega_{0}=\gamma_{0} \lambda b N_{w}<N_{w}$. By step 3, together with steps 1.1 and 1.2, it follows that $\gamma_{t}<\bar{\gamma}_{0}=\frac{1}{\lambda b}$ for all $t \leqq \tau$, and $\gamma_{t}=\bar{\gamma}_{0}=\frac{1}{\lambda b}$ for all $t, T-1 \geqq t \geqq \tau+1$. Therefore $L y_{t}=L A^{-1} \omega_{t}<N_{w}$, all $t \leqq \tau$, whereas $L y_{t}=L A^{-1} \omega_{t}=N_{w}$, all $t, T-1 \geqq t \geqq \tau+1$. Hence, for all $\eta \in \mathcal{N}_{w}$, assign a vector $\xi^{\eta}$ such that $z_{t}^{\eta}=\widehat{z}_{t}^{\eta}=\gamma_{t} \lambda b$ and $c_{t}^{\eta}=b$, all $t \leqq \tau$, and $z_{t}^{\eta}=1$ and $c_{t}^{\eta}=\frac{1}{p_{\beta}}$, all $t, T-1 \geqq t \geqq \tau+1$. Noting that $p_{\beta}=L\left[1-\left(1+\pi_{\beta}\right) A\right]^{-1}$ and $\pi_{\beta}>\pi^{\prime}$ imply $\frac{1}{p_{\beta}}>b$, it follows that $\xi^{\eta}$ solves $M P^{\eta}$, for all $\eta \in \mathcal{N}_{w}$. Hence parts (ii) and (v) of Definition 1 are met.

6. (Final goods market.) Consider first all periods $t \leqq \tau$. By construction at all $t, t \leqq \tau, c_{t}^{\eta}=b$, all $\eta \in \mathcal{N}_{w}$, and $c_{t}^{\nu}+s_{t}^{\nu}=\pi_{t} \omega_{t}^{\nu}$, all $\nu \in \mathcal{N}_{c}$. Therefore $c_{t}^{c}+s_{t}+c_{t}^{w}=\pi_{t} \omega_{t}+N_{w} b$, and substituting for $\pi_{t}=\widetilde{\pi} \frac{\left(\gamma_{t}-1\right)}{\gamma_{t}}=\frac{1-A}{A} \frac{\left(\gamma_{t}-1\right)}{\gamma_{t}}$ and $\omega_{t}=\gamma_{t} N_{w} A(1-A)^{-1} b$, one obtains $c_{t}^{c}+s_{t}+c_{t}^{w}=\gamma_{t} N_{w} b$. Because $(1-A) y_{t}=(1-A) A^{-1} \omega_{t}=\gamma_{t} N_{w} b$, it follows that $(1-A) y_{t}=c_{t}^{c}+s_{t}+c_{t}^{w}$, all $t, t \leqq \tau$. Consider next periods $t, T-1 \geqq t \geqq \tau+1$. By construction, at all $t, T-1 \geqq t \geqq \tau+1, c_{t}^{\eta}=1 / p_{\beta}$, all $\eta \in \mathcal{N}_{w}$, and $c_{t}^{\nu}+s_{t}^{\nu}=\pi_{\beta} \omega_{t}^{\nu}$, all $\nu \in \mathcal{N}_{c}$, and so $c_{t}^{c}+s_{t}+c_{t}^{w}=\pi_{\beta} \omega_{t}+\frac{N_{w}}{p_{\beta}}$. By substituting for $\pi_{\beta}$ and $p_{\beta}$, and noting that at the proposed path $\gamma_{t}=1 / \lambda b$, one obtains $c_{t}^{c}+s_{t}+c_{t}^{w}=N_{w} b / \lambda b$. Because $(1-A) y_{t}=(1-A) A^{-1} \omega_{t}=\gamma_{t} N_{w} b=N_{w} b / \lambda b$, it follows that $(1-A) y_{t}=c_{t}^{c}+s_{t}+c_{t}^{w}$, all $t \geqq \tau+1$. Therefore, Definition 1(iii) is met.

\section{Proof of Theorem 7:}

Proof. 1. Consider the sequence of profit rates $\left\{\pi_{t}\right\}_{t=0}^{T-1}$. Since $\gamma_{0}>1, \pi_{0} \in(0, \widetilde{\pi})$. Moreover, at all $t, \pi_{t}<\tilde{\pi}$ implies $\pi_{t+1}>\pi_{t}$. Therefore given $\beta\left[1+\widetilde{\pi} \frac{\left(\gamma_{0}-1\right)}{\gamma_{0}}\right]=\beta\left(1+\pi_{0}\right) \geqq 1$, it follows that $\pi_{t}>\pi_{\beta}$, all $t>0$. Finally, we prove that if $\pi_{0}=\tilde{\pi} \frac{\left(\gamma_{0}-1\right)}{\gamma_{0}}, \pi_{t+1}=\frac{\pi_{t}(1+\widetilde{\pi})}{\left(1+\pi_{t}\right)}$, all $t, T-2 \geqq t \geqq 0$, and $\gamma_{t+1}=\left(1+\pi_{t}\right) \gamma_{t}$, all $t, T-2 \geqq t \geqq 0$, then $\pi_{t}=\tilde{\pi} \frac{\left(\gamma_{t}-1\right)}{\gamma_{t}}$, all $t>0$. To see this, suppose the result holds for any $t>0$. Then $\pi_{t+1}=\frac{\pi_{t}(1+\widetilde{\pi})}{\left(1+\pi_{t}\right)}$ and $\gamma_{t+1}=\left(1+\pi_{t}\right) \gamma_{t}$ imply $\pi_{t+1}=\frac{\pi_{t} \gamma_{t}(1+\widetilde{\pi})}{\gamma_{t+1}}$. Because $\pi_{t}=\tilde{\pi} \frac{\left(\gamma_{t}-1\right)}{\gamma_{t}}$, the latter expression becomes $\pi_{t+1}=\frac{\widetilde{\pi}\left(\gamma_{t}-1\right)(1+\widetilde{\pi})}{\gamma_{t+1}}$, and the desired result follows noting that $\gamma_{t+1}=\left(1+\pi_{t}\right) \gamma_{t}$ and $\pi_{t}=\tilde{\pi} \frac{\left(\gamma_{t}-1\right)}{\gamma_{t}}$ imply $\gamma_{t+1}-1=(1+\tilde{\pi})\left(\gamma_{t}-1\right)$, as required.

2. (Optimal $\xi^{\nu}$; reproducibility.) By step $1, \pi_{t}>\pi_{\beta}$, all $t>0$. Therefore, by Theorem 5 , for all $\nu \in \mathcal{N}_{c}$, the vector $\xi^{\nu}$ with $y_{t}^{\nu}=A^{-1} \omega_{t}^{\nu}$, all $t ; \omega_{t+1}^{\nu}=\left(1+\pi_{t}\right) \omega_{t}^{\nu}$ and $c_{t}^{\nu}=0$, all $t, T-2 \geqq t \geqq 0$; $\omega_{T}^{\nu}=\omega_{0}^{\nu}$; and $c_{T-1}^{\nu}=\left(1+\pi_{T-1}\right) \omega_{T-1}^{\nu}-\omega_{0}^{\nu}$ solves $M P^{\nu}$. Hence parts (i) and (vi) of Definition 1 are met.

3. (Capital market) Because $y_{t}^{\nu}=A^{-1} \omega_{t}^{\nu}$, all $t$ and all $\nu \in \mathcal{N}_{c}$, then $y_{t}=A^{-1} \omega_{t}$, all $t$, and Definition 1(iv) is satisfied.

4. (Labor market; optimal $\xi^{\eta}$ ) By step $3, L_{t} y_{t}=L_{t} A^{-1} \omega_{t}=\gamma_{t} \lambda_{t} b N_{w}$, all $t$. By (A2), $L_{t+1}=$ $\delta L_{t}$, all $t, T-2 \geqq t \geqq 0$, and by step $3 y_{t+1}=y_{t}\left(1+\pi_{t}\right)$, all $t, T-2 \geqq t \geqq 0$. Hence, $L_{t+1} y_{t+1}=\delta\left(1+\pi_{t}\right) L_{t} y_{t}$, all $t, T-2 \geqq t \geqq 0$. Therefore, since $L_{0} y_{0}=L_{0} A^{-1} \omega_{0}=\gamma_{0} \lambda_{0} b N_{w} \leqq N_{w}$ and $\delta(1+\tilde{\pi}) \leqq 1$ by assumption, and $\pi_{t}<\tilde{\pi}$, all $t$, it follows that $L_{t} y_{t} \leqq N_{w}$, all $t$, and $L_{t} y_{t}<N_{w}$, all $t>0$. Then, for all $\eta \in \mathcal{N}_{w}$, let $\xi^{\eta}$ be defined by $z_{t}^{\eta}=\widehat{z}_{t}^{\eta}=\gamma_{t} \lambda_{t} b$ and $c_{t}^{\eta}=b$, all $t$. Noting that $\gamma_{t} \lambda_{t} b \leqq 1$, all $t$, and $p_{t}=L_{t}\left[1-\left(1+\pi_{t}\right) A\right]^{-1}=L_{t}\left[1-\left(1+\frac{1-A}{A} \frac{\left(\gamma_{t}-1\right)}{\gamma_{t}}\right) A\right]^{-1}=\gamma_{t} \lambda_{t}$, all $t$, it follows that $\xi^{\eta}$ solves $M P^{\eta}$, all $\eta \in \mathcal{N}_{w}$. Therefore parts (ii) and (v) of Definition 1 are met.

5. (Final goods market) At the proposed path, $c_{t}^{w}=N_{w} b$ and $c_{t}^{c}+s_{t}=\pi_{t} \omega_{t}$, all $t$, and substituting for $\pi_{t}=\tilde{\pi} \frac{\left(\gamma_{t}-1\right)}{\gamma_{t}}=\frac{1-A}{A} \frac{\left(\gamma_{t}-1\right)}{\gamma_{t}}$ and $\omega_{t}=\gamma_{t} N_{w} A(1-A)^{-1} b$, one obtains $c_{t}^{c}+s_{t}+c_{t}^{w}=$ $\gamma_{t} N_{w} b$, all $t$. Because $(1-A) y_{t}=(1-A) A^{-1} \omega_{t}=\gamma_{t} N_{w} b$, all $t$, Definition 1(iii) is satisfied. 


\section{References}

[1] Acemoglu, D. (2002). "Directed Technical Change", Review of Economic Studies, 69, 781-809.

[2] Acemoglu, D. (2002). "Technical Change, Inequality, and the Labor Market", Journal of Economic Literature, XL, 7-72.

[3] Acemoglu, D. (2003). "Labor- and Capital-Augmenting Technical Change", Journal of the European Economic Association, 1, 1-37.

[4] Allegretto, S.A. (2011). "The State of Working America's Wealth", EPI Briefing Paper, N. 292, March 23, 2011.

[5] Banerjee, A.V. and Newman, A.F. (1993). "Occupational Choice and the Process of Development", Journal of Political Economy, 101, 274-298.

[6] Banerjee, A.V. and Newman, A.F. (1993). "Risk-Bearing and the Theory of Income Distribution", Review of Economic Studies, 58, 211-235.

[7] Barone, G. and Mocetti,S. (2016). "Intergenerational mobility in the very long run: Florence 1427-2011", Bank of Italy, WP 1060, April 2016.

[8] Basu, D. (2009). "A simple test of the neoclassical view of production, technical change and distribution", Metroeconomica, 61, 593-621.

[9] Becker, G., Murphy, K. and Werning, I. (2005). "The Equilibrium Distribution of Income and the Market for Status", Journal of Political Economy, 113, 282-301.

[10] Bénabou, R. (1996). "Inequality and Growth", in (Bernanke, B.S. and Rotemberg, J.J., eds.) (1996) NBER Macroeconomics Annual 1996, Volume 11. Cambridge: MIT Press.

[11] Bénabou, R. and Tirole, J. (2006). "Belief in a Just World and Redistributive Politics", Quarterly Journal of Economics, 121, 699-746.

[12] Bidard, C. (1999). "An Algorithmic Theory of the Choice of Techniques", Econometrica, 58, 839-859.

[13] Böhm, V. and Kaas, L. (2000). "Differential savings, factor shares, and endogenous growth cycles", Journal of Economic Dynamics and Control, 24, 965-980.

[14] Devine, J. and Dymski, G. (1991). "Roemer's 'General' Theory of Exploitation is a Special Case", Economics and Philosophy, 7, 235-275.

[15] Dosi, G., Pereira, M.C., Roventini, A., and Virgillito, M.E. (2017). "When more flexibility yields more fragility: The microfoundations of Keynesian aggregate unemployment", Journal of Economic Dynamics and Control, 81, 162-186.

[16] Fleurbaey, M. (2014). "The facets of exploitation", Journal of Theoretical Politics, 26, 653-676.

[17] Foley, D.K. and Michl, T.R. (1999). Growth and distribution, Cambridge: Harvard University Press.

[18] Galor, O. and Zeira, J. (1993). "Income Distribution and Macroeconomics", Review of Economic Studies, 60, 35-52.

[19] Galor, O. and Tsiddon, D. (1997). "Technological Progress, Mobility, and Economic Growth", American Economic Review, 87, 363-382. 
[20] Genicot, G. and Ray, D. (2017). "Aspirations and Inequality", Econometrica, 85, 489-519.

[21] Greenstone, M., Looney, A., Patashnik, J. and Yu, M. (2013). "Thirteen Economic Facts about Social Mobility and the Role of Education", Brookings Institution, June 26, 2013.

[22] Hahnel, R. (2006). "Exploitation: A Modern Approach", Review of Radical Political Economics, 38, 175-192.

[23] Hahnel, R. (2007). Income Distribution and Environmental Sustainability. London: Routledge.

[24] Kaldor, N. (1956). "Alternative theories of distribution", Review of Economic Studies, 23, 83-100.

[25] Kalecki, M. (1943 [1990]). "Political aspects of full employment" reprinted in (Osiatynski, J., ed.) (1990) Collected Works of Michal Kalecki, Volume I: Capitalism: Business Cycles and Full Employment. Oxford: Clarendon Press.

[26] Kaplan, G. and Violante, G. (2014), "A Model of the Consumption Response to Fiscal Stimulus Payments", Econometrica, 82, 1199-1239.

[27] Karabarbounis, L. and Neiman, B. (2014). "The global decline of the labor share", Quarterly Journal of Economics 129, 61-103.

[28] Kennedy, C. (1964). "Induced bias in innovation and the theory of distribution", Economic Journal, 74, 541-547.

[29] Krueger, D., Mitman, K., and Perri, F. (2016). "Macroeconomics and Household Heterogeneity", in (Taylor, J.B. and Uhlig, H., eds.) Handbook of Macroeconomics, Amsterdam: North Holland.

[30] Kurz, H.D. and Salvadori, N. (1995). Theory of Production, Cambridge: Cambridge University Press.

[31] Loury, G. (1981). "Intergenerational transfers and the distribution of earnings", Econometrica, $49,843-867$.

[32] Malinvaud, E. (1972). Lectures on Microeconomic Theory, Advanced Textbooks in Economics, Revised Edition, Amsterdam: North Holland.

[33] Mandler, M. (1999). "Sraffian Indeterminacy in General Equilibrium", Review of Economic Studies, 66, 693-711.

[34] Maniquet, F. (2002). "A study of proportionality and robustness in economies with a commonly owned technology", Review of Economic Design, 7, 1-15.

[35] Matsuyama, K. (2004). "Financial Market Globalization, Symmetry-Breaking, and Endogenous Inequality of Nations", Econometrica, 72, 853-884.

[36] Mookherjee, D. and Ray, D. (2017). "Capital and Inequality in the Long Run", mimeo, Boston University and NYU.

[37] Morishima, M. (1969). Theory of Economic Growth. Oxford: Oxford University Press.

[38] Morishima, M. (1974). "Marx in the light of modern economic theory", Econometrica, 42, 611-632.

[39] Moulin, H. (1990). "Joint Ownership of a Convex Technology: Comparison of Three Solutions", Review of Economic Studies, 57, 439-452. 
[40] Okishio, N. (1963). "A Mathematical Note on Marxian Theorems", Weltwirtschaftlisches Archiv, 91, 287-99.

[41] Pasinetti, L. (1962). "Rate of profit and income distribution in relation to the rate of economic growth", Review of Economics Studies, 29, 267-279.

[42] Patriarca, F. and Vona, F. (2013). "Structural change and income distribution: An inverted-U relationship", Journal of Economic Dynamics and Control, 37, 1641-1658.

[43] Pigou, A.C. (1920). The Economics of Welfare, London: Macmillan.

[44] Piketty, T. (2014). Capital in the Twenty-First Century, Cambridge, MA: Harvard University Press.

[45] Piketty, T. and Zucman, G. (2014). "Capital is back: wealth-income ratios in rich countries 1700-2010", Quarterly Journal of Economics, 129, 1255-1310.

[46] Ray, D. and Esteban, J. (2017). "Conflict and Development", Annual Review of Economics, 9, 263-93.

[47] Ray, D. and Robson, A. (2012). "Status, Intertemporal Choice and Risk-Taking", Econometrica, 80, 1505-1531.

[48] Rawls, J. (1971). A Theory of Justice, Cambridge: Harvard University Press.

[49] Robinson, J.V. (1933). The Economics of Imperfect Competition, London: Macmillan.

[50] Roemer, J.E. (1980). "A General Equilibrium Approach to Marxian Economics", Econometrica, 48, 505-530.

[51] Roemer, J.E. (1981). Analytical Foundations of Marxian Economic Theory, Cambridge: Harvard University Press.

[52] Roemer, J.E. (1982). A General Theory of Exploitation and Class, Cambridge: Harvard University Press.

[53] Roemer, J.E. (1982). "Origins of Exploitation and Class: Value Theory of Pre-Capitalist Economy", Econometrica, 50, 163-192.

[54] Roemer, J.E. (1994). Egalitarian Perspectives: Essays in Philosophical Economics, Cambridge: Cambridge University Press.

[55] Roemer, J.E. (2010). "Kantian equilibrium", Scandinavian Journal of Economics, 112, 1-24.

[56] Roemer, J.E. and J. Silvestre (1993): "The proportional solution for economies with both private and public ownership," Journal of Economic Theory, 59, 426-444.

[57] Sen, A.K. (1999). "The Possibility of Social Choice", American Economic Review, 89, 349-378.

[58] Shell, K. (1967). "A Model of Inventive Activity and Capital Accumulation", in (Shell, K. ed.) Essays on the Theory of Optimal Economic Growth. Cambridge: MIT Press.

[59] Skillman, G.L. (2017). "Marx's Capital Through the Lens of Roemer's General Theory (and Vice-Versa)", Social Choice and Welfare, 49, 423-443.

[60] Solow, R. M. (1956) "A Contribution to the Theory of Economic Growth", The Quarterly Journal of Economics, 70, 65-94 
[61] Tavani, D. and Zamparelli, L. (2015). "Endogenous technical change, employment and distribution in the Goodwin model of the growth cycle", Studies in Nonlinear Dynamics 83 Econometrics, 19, 209-226.

[62] Veneziani, R. (2007). "Exploitation and Time", Journal of Economic Theory, 132, 189-207.

[63] Veneziani, R. and Yoshihara, N. (2015). "Exploitation in economies with heterogeneous preferences, skills and assets: An axiomatic approach", Journal of Theoretical Politics, 27, 8-33.

[64] Veneziani, R. and Yoshihara, N. (2017). "One million miles to go: taking the axiomatic road to defining exploitation", Cambridge Journal of Economics, 41, 1607-1626.

[65] Veneziani, R. and Yoshihara, N. (2017). "Globalisation and Inequality in a Dynamic Economy: An Axiomatic Analysis of Unequal Exchange", Social Choice and Welfare, 49, 445-468.

[66] von Neumann, J. (1945-1946). "A Model of General Equilibrium", Review of Economic Studies $13,1-9$.

[67] Yoshihara, N. (2010). "Class and Exploitation in General Convex Cone Economies", Journal of Economic Behavior \& Organization, 75, 281-296.

[68] Yoshihara, N. and Veneziani, R. (2018). "Technical Change, Capital Accumulation, and Factor Income Distribution", mimeo.

[69] Yoshihara, N. and Veneziani, R. (2018). "The theory of exploitation as the unequal exchange of labour", forthcoming in Economics and Philosophy.

[70] Yoshihara, N. and Kwak, S-H. (2018). "Sraffian Indeterminacy in General Equilibrium Revisited", mimeo. 